\title{
Preparation and light-mediated distribution of motional state entanglement
}

\author{
A.S. Parkins \\ Department of Physics, University of Auckland, Private Bag 92019, Auckland, New Zealand \\ E. Larsabal \\ Département de Physique de l'Ecole Normale Supérieure, 24 rue Lhomond, F-75231, Paris Cedex 05, France
}

\begin{abstract}
We describe and analyse numerically schemes (i) for entangling orthogonal motional modes of one or a few harmonicallytrapped atoms or ions, and (ii) for transferring the entanglement from one of these local modes to a distant trapped atom (or atoms) via a light-mediated quantum state transfer procedure proposed in previous work [A.S. Parkins and H.J. Kimble, J. Opt. B: Quantum Semiclass. Opt. 1, 496 (1999)]. Possibilities arising from these schemes include the generation of an Einstein-Podolsky-Rosen state in the positions and momenta of distantly-separated trapped atoms and the preparation of delocalized mesoscopic vibrational states.
\end{abstract}

PACS numbers: 03.67.Hk, 32.80.Lg, 42.50.-p

\section{INTRODUCTION}

In addition to being of fundamental interest in physics, quantum entanglement is an essential resource for quantum information processing and distribution. Popular candidates for experimental investigation in this context include trapped atoms, cavity quantum electrodynamics (cavity QED), and nonclassical light fields, using which a variety of impressive quantum state manipulations have now been demonstrated, including elementary quantum logic operations 114, preparation of quantummechanically entangled pairs [5] and quadruplets 8] of atoms, and quantum teleportation 9911.

In recent work we have proposed a scheme that offers the possibility of combining the features of trapped atoms and nonclassical light fields in a distributed network [12]; in particular, a scheme that enables motional quantum states to be coupled to propagating (nonclassical) light fields via interactions in cavity QED. This coupling enables the deterministic generation and distribution of quantum entanglement between different atoms and/or different light fields. One particular example we have considered is the preparation of an Einstein-PodolskyRosen (EPR) state in the positions and momenta of a pair of distantly-separated trapped atoms [13], which in turn leads to a scheme for the teleportation of motional quantum states 14].

For the proposals presented in 13,14 entanglement of the atoms' motional states is achieved through the transfer of entanglement from the quantum-correlated output light fields from a nondegenerate parametric amplifier. However, as pointed out in [12], the trapped-atom cavity QED setup can itself act as a source of nonclassical light fields; nonclassical motional states (prepared by some means independent of the atom-cavity coupling) can be transferred to the propagating output field from the cavity, which can in turn be coupled to the motion of a second atom confined inside another, distant cavity. The potential of such a trapped-atom nonclassical light source is underscored by the impressive control with which the motional quantum states of single trapped atoms can be controlled and, more especially, by the variety of nonclassical motional states that have in fact been prepared experimentally 15 18.

The purpose of the present paper, then, is to expand upon this idea and present schemes whereby "local" entanglement of orthogonal motional modes of a single atom (or of a few atoms) trapped inside an optical cavity is transformed, via propagating light fields, into "nonlocal" entanglement of the motional modes of distantlyseparated atoms. In this way, the need for nonlinear (quantum) optical devices, such as nondegenerate parametric amplifiers, is eliminated, and all of the desired operations are achieved using only trapped-atom cavity QED configuratons.

In Section II we present a relatively simple example of a scheme for the manipulation of the motional state of a trapped atom (or atoms) in two dimensions using only (external) laser fields. This scheme allows entanglement to be established between two orthogonal modes and the results of some numerical simulations for the case of a two-mode "squeezed" state of the motion are presented. Section III describes the trapped-atom cavity QED system that facilitates the motion-light coupling and is, of course, central to our proposals; through this coupling, the quantum state (i.e., the entanglement) of one of the two relevant motional modes can be "mapped onto" a propagating light field and transferred to a distant site. We examine this system in some detail, presenting numerical calculations that further the original analysis of 12 , looking more closely, for example, at the Lamb-Dicke (tight confinement) assumption involved in the model. Having described means for preparing "local" entanglement of motional modes and for transferring motional quantum states between distant atoms, the preparation of distributed "nonlocal" motional state entanglement follows naturally, and in Section IV we discuss several examples, such as the position-momentum EPR state and delocalized mesoscopic states of the motion. 


\section{ENTANGLING ORTHOGONAL MOTIONAL MODES AT ONE LOCATION}

Schemes for coupling and manipulating orthogonal motional modes in a single-atom trap have been put forward by a variety of authors 19 26, typically involving stimulated-Raman transitions between internal atomic states. Here, we describe one such scheme, outlining how certain effective interaction Hamiltonians (of particular interest to us) may be realized and presenting some numerical simulations. This scheme involves only a single internal atomic level (after adiabatic elimination of the excited internal state) and generalizes to two dimensions a technique already used in ion-trap experiments to prepare, for example, one-dimensional squeezed states of the motion 15 .

\section{A. Coupling orthogonal motional modes of a single trapped atom}

In this scheme, counterpropagating laser beams aligned (in our case) in the $x-z$ plane and coupled to the same internal atomic transition induce a coupling between orthogonal ( $x$ and $z$ ) motional modes which, depending on the detuning between the two laser fields, may take the form of a linear mixer or a nondegenerate parametric amplifier.

The physical setup is depicted in Fig. 1(a). Mathematically, the situation is described by the Hamiltonian

$$
\begin{aligned}
\hat{H}(t)= & \hbar \Delta_{01} \hat{\sigma}_{+} \hat{\sigma}_{-}+\sum_{j=x, z} \hbar \nu_{j}\left(\hat{b}_{j}^{\dagger} \hat{b}_{j}+1 / 2\right) \\
& +i \hbar\left[E_{\mathrm{L}}^{*}(\hat{x}, \hat{z}, t) \hat{\sigma}_{-}-E_{\mathrm{L}}(\hat{x}, \hat{z}, t) \hat{\sigma}_{+}\right],
\end{aligned}
$$

where $\nu_{x}$ and $\nu_{z}$ are the harmonic oscillation frequencies along the $x$ and $z$ axes of the trap, $\hat{b}_{j}$ are annihilation operators for the quantized atomic motion, and $\hat{\sigma}_{-}=$ $|g\rangle\langle e|$ is the atomic lowering operator for the $|g\rangle \leftrightarrow|e\rangle$ transition. The total field incident on the atom is given by

$$
\begin{aligned}
E_{\mathrm{L}}(\hat{x}, \hat{z}, t) & =E_{1}(\hat{x}, \hat{z}, t)+E_{2}(\hat{x}, \hat{z}, t) \\
& =\frac{\mathcal{E}}{\sqrt{2}}\left[e^{-i k(\alpha \hat{x}+\beta \hat{z})}+e^{i k(\alpha \hat{x}+\beta \hat{z})-i \delta_{21} t+i \phi}\right],
\end{aligned}
$$

where $\alpha$ and $\beta$ are determined by the angle of incidence in the $x-z$ plane and satisfy $\left(\alpha^{2}+\beta^{2}\right)^{1 / 2}=1$. The position operators are given by $\hat{x}=\left[\hbar /\left(2 m \nu_{x}\right)\right]^{1 / 2}\left(\hat{b}_{x}+\hat{b}_{x}^{\dagger}\right)$ and $\hat{z}=\left[\hbar /\left(2 m \nu_{z}\right)\right]^{1 / 2}\left(\hat{b}_{z}+\hat{b}_{z}^{\dagger}\right)$, and $k=2 \pi / \lambda$ is the wavenumber (taken to be the same for both fields). The detunings $\Delta_{01}$ and $\delta_{21}$ are given by $\Delta_{01}=\omega_{0}-\omega_{1}$ and $\delta_{21}=\omega_{2}-\omega_{1}$, where $\omega_{0}$ is the atomic transition frequency and $\omega_{1}$ and $\omega_{2}$ are the frequencies of the two counterpropagating fields.

With the assumption that $\Delta_{01}$ is large, the atomic excited state can be adiabatically eliminated and spontaneous emission neglected. In the Heisenberg equations of motion one makes the substitution

$$
\hat{\sigma}_{-} \simeq i \frac{E_{\mathrm{L}}(\hat{x}, \hat{z}, t)}{\Delta_{01}},
$$

which gives, for the motional mode operators,

$$
\begin{aligned}
\dot{\hat{b}}_{x, z} \simeq & -i \nu_{x, z} \hat{b}_{x, z}+\frac{2 i}{\Delta_{01}}\left[\hat{b}_{x, z},\left|E_{\mathrm{L}}(\hat{x}, \hat{z}, t)\right|^{2}\right] \\
= & -i \nu_{x, z} \hat{b}_{x, z} \\
& +\frac{2 \eta_{x, z}^{\prime} \mathcal{E}^{2}}{\Delta_{01}}\left[e^{-2 i k(\alpha \hat{x}+\beta \hat{z})+i \delta_{21} t-i \phi}-\text { h.c. }\right],
\end{aligned}
$$

where $\eta_{x}^{\prime}=\alpha \eta_{x}$ and $\eta_{z}^{\prime}=\beta \eta_{z}$, with $\eta_{x}=k\left(\hbar / 2 m \nu_{x}\right)^{1 / 2}$ and $\eta_{z}=k\left(\hbar / 2 m \nu_{z}\right)^{1 / 2}$.

Moving to a rotating frame with respect to the motion, i.e., defining $\hat{b}_{x, z}=\tilde{b}_{x, z} e^{-i \nu_{x, z} t}$, and making the LambDicke approximation, i.e., expanding the exponentials to first order in $\eta_{x, z}^{\prime}$, one derives

$$
\begin{aligned}
\dot{\tilde{b}}_{x} \simeq & \frac{2 \eta_{x}^{\prime} \mathcal{E}^{2}}{\Delta_{01}} e^{i \nu_{x} t}\left(e^{i \delta_{21} t-i \phi}-e^{-i \delta_{21} t+i \phi}\right) \\
& -\frac{4 i \eta_{x}^{\prime 2} \mathcal{E}^{2} e^{-i \phi}}{\Delta_{01}} e^{i\left(\nu_{x}+\delta_{21}\right) t}\left(\tilde{b}_{x} e^{-i \nu_{x} t}+\tilde{b}_{x}^{\dagger} e^{i \nu_{x} t}\right) \\
& -\frac{4 i \eta_{x}^{\prime} \eta_{z}^{\prime} \mathcal{E}^{2} e^{-i \phi}}{\Delta_{01}} e^{i\left(\nu_{x}+\delta_{21}\right) t}\left(\tilde{b}_{z} e^{-i \nu_{z} t}+\tilde{b}_{z}^{\dagger} e^{i \nu_{z} t}\right) \\
& -\frac{4 i \eta_{x}^{\prime 2} \mathcal{E}^{2} e^{i \phi}}{\Delta_{01}} e^{i\left(\nu_{x}-\delta_{21}\right) t}\left(\tilde{b}_{x} e^{-i \nu_{x} t}+\tilde{b}_{x}^{\dagger} e^{i \nu_{x} t}\right) \\
& -\frac{4 i \eta_{x}^{\prime} \eta_{z}^{\prime} \mathcal{E}^{2} e^{i \phi}}{\Delta_{01}} e^{i\left(\nu_{x}-\delta_{21}\right) t}\left(\tilde{b}_{z} e^{-i \nu_{z} t}+\tilde{b}_{z}^{\dagger} e^{i \nu_{z} t}\right)
\end{aligned}
$$

(and similarly for $\dot{\tilde{b}}_{z}$ ). This equation contains a variety of terms oscillating at a variety of different frequencies. With a judicious choice of the detuning $\delta_{21}$ between the two laser fields, a particular term can have its time dependence removed, whereas the other (oscillating) terms can, provided the trap frequencies are sufficiently large (and different), be neglected in a rotating-wave approximation. We now consider two cases of interest to us.

\section{Linear mixing: rotation of the motional state}

To realize a linear mixing of the $x$ and $z$ modes (i.e., a beamsplitter-type interaction), we choose the detuning between the two incident laser fields to be $\delta_{21}=\nu_{x}-\nu_{z}$. With this choice, only one term in (6) is "stationary", while all of the other terms retain oscillatory factors. Provided the frequencies $\nu_{x}, \nu_{z}$, and $\left|\nu_{x}-\nu_{z}\right|$ are large compared to the effective coupling strength between the two modes, these oscillatory terms can be dropped in a rotating-wave approximation, leaving

$$
\dot{\tilde{b}}_{x, z} \simeq-i\left(\frac{4 \eta_{x}^{\prime} \eta_{z}^{\prime} \mathcal{E}^{2} e^{ \pm i \phi}}{\Delta_{01}}\right) \tilde{b}_{z, x}
$$

The effective interaction Hamiltonian is thus 


$$
\tilde{H}_{\text {mix }}=\hbar \chi\left(\tilde{b}_{x}^{\dagger} \tilde{b}_{z} e^{i \phi}+\text { h.c. }\right),
$$

with the interaction strength given by

$$
\chi=\frac{4 \eta_{x}^{\prime} \eta_{z}^{\prime} \mathcal{E}^{2}}{\Delta_{01}} .
$$

The physical process corresponding to this situation is illustrated in Fig. 1(b). Particular cases of interest are those in which the interaction is turned on for a time $T$ such that $\chi T=\pi / 4$ or $\chi T=\pi / 2$ (we assume rectangular pulse shapes); the first case effects an operation equivalent to a 50/50 beamsplitter, while in the second case the result is a complete exchange of states between the two modes, i.e., for $\phi=\pi / 2$ one finds $\tilde{b}_{x}(T)=-\tilde{b}_{z}(0)$ and $\tilde{b}_{z}(T)=\tilde{b}_{x}(0)$.

Note also that, given suitable (unentangled) initial states of the $x$ and $z$ modes (prepared by some other means), one can generate entanglement with the beamsplitter $(\chi T=\pi / 4)$ operation. For example, an initial product of number states,

$$
|\psi(0)\rangle=\left|N_{x}\right\rangle_{x} \otimes\left|N_{z}\right\rangle_{z},
$$

is transformed via a 50/50 beamsplitter operation into an entangled state of the form

$$
|\psi(T)\rangle=\sum_{n=0}^{N} d_{n}|N-n\rangle_{x} \otimes|n\rangle_{z},
$$

where $N=N_{x}+N_{z}$ and $\left\{d_{n}\right\}$ are certain coefficients.

\section{Two-mode squeezing of the motional state}

Alternatively, we may choose $\delta_{21}=\nu_{x}+\nu_{z}$, in which case, following the same assumptions as above, one derives

$$
\dot{\tilde{b}}_{x, z} \simeq-i\left(\frac{4 \eta_{x}^{\prime} \eta_{z}^{\prime} \mathcal{E}^{2} e^{ \pm i \phi}}{\Delta_{01}}\right) \tilde{b}_{z, x}^{\dagger}
$$

corresponding to

$$
\tilde{H}_{\mathrm{sq}}=\hbar \chi\left(\tilde{b}_{x}^{\dagger} \tilde{b}_{z}^{\dagger} e^{i \phi}+\text { h.c. }\right),
$$

with $\chi$ as before. The physical process corresponding to this situation is illustrated in Fig. 1(c); it is of course a parametric amplification process, which leads to "twomode squeezing". Taking $\phi=-\pi / 2$, the state produced after an interaction time $T$, given the modes are initially in their ground vibrational levels (achieved, for example, by sideband cooling), is

$$
\begin{aligned}
\left|\psi_{\mathrm{sq}}(r)\right\rangle & =S_{x z}(r)|0\rangle_{x} \otimes|0\rangle_{z} \\
& =[\cosh (r)]^{-1} \sum_{m=0}^{\infty}[-\tanh (r)]^{m}|m\rangle_{x} \otimes|m\rangle_{z}
\end{aligned}
$$

where $|m\rangle_{x, z}$ are Fock states of the motional modes and $S_{x z}(r)$ is the two-mode squeezing operator 27,

$$
S_{x z}(r)=\exp \left[r\left(\tilde{b}_{x} \tilde{b}_{z}-\tilde{b}_{x}^{\dagger} \tilde{b}_{z}^{\dagger}\right)\right],
$$

with $r=\chi T$. The entanglement between modes that is generated by this process is best expressed in terms of position and momentum variables. In particular, one can show for the state (14) that the Wigner function in these variables is

$$
\begin{aligned}
& W\left(\tilde{x}, \tilde{p}_{x} ; \tilde{z}, \tilde{p}_{z}\right) \\
& =\frac{4}{\pi^{2}} \exp \left\{-\left[(\tilde{x}+\tilde{z})^{2}+\left(\tilde{p}_{x}-\tilde{p}_{z}\right)^{2}\right] e^{+2 r}\right\} \\
& \quad \times \exp \left\{-\left[(\tilde{x}-\tilde{z})^{2}+\left(\tilde{p}_{x}+\tilde{p}_{z}\right)^{2}\right] e^{-2 r}\right\} \\
& \sim C \delta(\tilde{x}+\tilde{z}) \delta\left(\tilde{p}_{x}-\tilde{p}_{z}\right) \quad \text { for large } r,
\end{aligned}
$$

with $C$ a constant 27,28]. This, of course, corresponds to the original state considered by Einstein, Podolsky, and Rosen in their famous gedanken experiment [29], although, in this particular situation the position and momentum variables belong to the same particle. However, as we will describe below, through our scheme for motionlight coupling we are able to distribute this entanglement between two distantly-separated atoms.

\section{B. Numerical analysis}

Before describing the scheme for distribution of motional state entanglement, we consider the results of some numerical simulations of the state manipulation schemes proposed above. By numerical simulation, we mean direct integration of the Schrödinger equation

$$
\frac{\partial}{\partial t}|\psi(t)\rangle=\frac{1}{i \hbar} \hat{H}_{\mathrm{ad}}(t)|\psi(t)\rangle
$$

with $\hat{H}_{\text {ad }}(t)$ given by (omitting the constant ground state vibrational energy)

$$
\hat{H}_{\mathrm{ad}}(t)=\hbar \nu_{x} \hat{b}_{x}^{\dagger} \hat{b}_{x}+\hbar \nu_{z} \hat{b}_{z}^{\dagger} \hat{b}_{z}-\hbar \frac{2\left|E_{\mathrm{L}}(\hat{x}, \hat{z}, t)\right|^{2}}{\Delta_{01}}
$$

which is the form one obtains upon adiabatically eliminating the atomic excited state. Truncated number state bases are used to describe the harmonic oscillator modes describing the two-dimensional motion.

We have focussed in our work on the two-mode squeezing, or EPR entanglement, operation of the previous subsection, determining in particular suitable parameter regimes for the efficient implementation of the scheme (requirements for the linear mixing scheme are basically the same). As a measure of how well the scheme works, we consider the fidelity

$$
F=\left|\left\langle\psi_{\mathrm{sq}}(r) \mid \psi(T)\right\rangle_{\mathrm{sim}}\right|^{2},
$$

where $|\psi(T)\rangle_{\text {sim }}$ is the simulated wave function after a time $T$, and $r=\chi T$ with $\chi$ given by (9). 
a. Results Sample values of $F$ are given in Table I for a number of different combinations of trapping frequencies and Lamb-Dicke parameters. As one can see, twomode squeezed states exhibiting significant degrees of entanglement $(r \gtrsim 1)$ can be prepared with high fidelity given sufficiently small Lamb-Dicke parameters and sufficiently large $\nu_{x}, \nu_{z}$, and $\left|\nu_{x}-\nu_{z}\right|$ (compared to $\left.\chi\right)$.

It becomes computationally prohibitive for us to consider values of $r$ much larger than $\sim 1.5$, owing to the large number state bases required to cover adequately the population distribution of the two-mode squeezed state. However, the results presented here suggest that, for example, a state with $r \simeq 2$ could be prepared with a fidelity exceeding 0.9 for physically reasonable parameters.

b. Experimental prospects If we take the example of trapped ${ }^{9} \mathrm{Be}^{+}$ions, $\eta_{x}^{\prime}=\eta_{z}^{\prime}=0.0707$ and $\nu_{x} / \nu_{z}=1 / 3$ correspond to actual trap frequencies $\nu_{x} / 2 \pi=11 \mathrm{MHz}$ and $\nu_{z} / 2 \pi=34 \mathrm{MHz}$ (which are essentially the frequencies achieved in the experiments of Monroe and coworkers [16]), and thus, for the example given in the table, to a value of the interaction strength $\chi / 2 \pi=22 \mathrm{kHz}$ and a timescale for the preparation of the entangled state on the order of $\chi^{-1} \simeq 7 \mu \mathrm{s}$. This should of course be much smaller than the timescale for motional state decoherence due to, for example, spontaneous emission, which we have neglected in our analysis. The rate of spontaneous emission events influencing the motional dynamics (i.e., incoherent scattering) can be estimated to be on the order of $\left(\gamma \eta_{x}^{2} \mathcal{E}^{2}\right) / \Delta_{01}^{2}$, where $\gamma^{-1}$ is the atomic excited state lifetime (see Appendix A, Part 1). Choosing $\gamma / \Delta_{01} \ll 1$, this rate can evidently be made much smaller than $\chi$.

\section{Two or more trapped ions: coupling collective and single ion modes}

Two or more ions confined in a linear ion trap [30] interact strongly through Coulomb repulsion and their motion along the axis of the trap is best described in terms of collective modes of vibration. Such collective modes have, of course, been fundamental to ion-trap quantum computer proposals 31. In the present context, we wish to point out that the schemes of Section II.A could also be used to couple and entangle orthogonal collective modes of a linear chain of trapped ions.

To illustrate this, we consider for simplicity just a pair of trapped ions confined in a harmonic potential and aligned along the $z$-axis, as illustrated in Fig. 2. Assuming very strong confinement in the $x$ direction (so that the motion along this axis can be regarded as independent of the $z$-axis collective motion), the quantized (two-dimensional) motion of the ions can be described by the Hamiltonian

$$
\hat{H}_{\mathrm{mot}}=\hbar \nu_{x} \sum_{j=1}^{2}\left(\hat{b}_{j x}^{\dagger} \hat{b}_{j x}+\frac{1}{2}\right)
$$

$$
+\hbar \nu_{0 z}\left(\hat{c}_{0 z}^{\dagger} \hat{c}_{0 z}+\frac{1}{2}\right)+\hbar \nu_{R z}\left(\hat{c}_{R z}^{\dagger} \hat{c}_{R z}+\frac{1}{2}\right),
$$

where $\hat{b}_{j x}, \hat{c}_{0 z}$, and $\hat{c}_{R z}$ are harmonic oscillator annihilation operators for single-ion motion along the $x$-axis, for the center-of-mass motion along the $z$-axis, and for the relative motion along the $z$-axis, respectively. The frequencies of the collective modes are related to the singleion mode frequency by $\nu_{0 z}=\nu_{z}$ and $\nu_{R z}=\sqrt{3} \nu_{z}$ [31, 32].

To couple the various modes via laser light, excitation of only a single ion is necessary, and this excitation takes the same form as described by (11) and (2); that is, we take

$$
\begin{aligned}
\hat{H}_{\text {ion-laser }} & =\hbar \Delta_{01} \hat{\sigma}_{+}^{(1)} \hat{\sigma}_{-}^{(1)} \\
& +i \hbar\left[E_{\mathrm{L}}^{*}\left(\hat{x}_{1}, \hat{z}_{1}, t\right) \hat{\sigma}_{-}^{(1)}-\text { h.c. }\right],
\end{aligned}
$$

with

$$
\begin{aligned}
& E_{\mathrm{L}}\left(\hat{x}_{1}, \hat{z}_{1}, t\right)= \\
& \quad \frac{\mathcal{E}}{\sqrt{2}}\left[e^{-i k\left(\alpha \hat{x}_{1}+\beta \hat{z}_{1}\right)}+e^{i k\left(\alpha \hat{x}_{1}+\beta \hat{z}_{1}\right)-i \delta_{21} t+i \phi}\right] .
\end{aligned}
$$

Expressed in terms of the collective mode operators, one has [33]

$$
e^{i k \hat{z}_{1}}=e^{i \eta_{0 z}\left(\hat{c}_{0 z}^{\dagger}+\hat{c}_{0 z}\right)} e^{i \eta_{R z}\left(\hat{c}_{R z}^{\dagger}+\hat{c}_{R z}\right)},
$$

with the collective mode Lamb-Dicke parameters given by

$$
\eta_{0 z}=\frac{\eta_{z}}{\sqrt{2}}, \quad \eta_{R z}=\frac{\eta_{z}}{\sqrt{2 \sqrt{3}}}
$$

As before, the atomic excited state is adiabatically eliminated and the position-dependent functions are expanded to first order in the various Lamb-Dicke parameters. The resulting Heisenberg equations of motion for the mode operators again display couplings between the modes with varying time dependencies, and again with a judicious choice of the detuning $\delta_{21}$ one can "select" a particular coupling (neglecting the others in a rotating-wave approximation), provided the frequencies $\nu_{x}, \nu_{0 z}$, and $\nu_{R z}$ are sufficiently large and different. The effective interaction Hamiltonians one can realize are thus (in the rotating frame)

$$
\tilde{H}_{\mathrm{eff}}= \begin{cases}\hbar \chi_{0}\left(\tilde{b}_{1 x}^{\dagger} \tilde{c}_{0 z} e^{i \phi}+\text { h.c. }\right), & \delta_{21}=\nu_{x}-\nu_{0 z} \\ \hbar \chi_{0}\left(\tilde{b}_{1 x}^{\dagger} \tilde{c}_{0 z}^{\dagger} e^{i \phi}+\text { h.c. }\right), & \delta_{21}=\nu_{x}+\nu_{0 z} \\ \hbar \chi_{R}\left(\tilde{b}_{1 x}^{\dagger} \tilde{c}_{R z} e^{i \phi}+\text { h.c. }\right), & \delta_{21}=\nu_{x}-\nu_{R z} \\ \hbar \chi_{R}\left(\tilde{b}_{1 x}^{\dagger} \tilde{c}_{R z}^{\dagger} e^{i \phi}+\text { h.c. }\right), & \delta_{21}=\nu_{x}+\nu_{R z}\end{cases}
$$

with

$$
\chi_{0}=\frac{4 \eta_{x}^{\prime} \eta_{0 z}^{\prime} \mathcal{E}^{2}}{\Delta_{01}}, \quad \chi_{R}=\frac{4 \eta_{x}^{\prime} \eta_{R z}^{\prime} \mathcal{E}^{2}}{\Delta_{01}}
$$


and $\eta_{x}^{\prime}=\alpha \eta_{x}, \eta_{0 z}^{\prime}=\beta \eta_{0 z}, \eta_{R z}^{\prime}=\beta \eta_{R z}$. So, one again realizes linear mixers and parametric amplifiers, only now one of the modes is a collective mode for the two ions. With the addition of more ions, one introduces more collective modes, but the frequencies of these modes remain incommensurate and (for large $\nu_{z}$ ) well-separated [32], implying that the above working could be readily generalized to three or more ions.

\section{COUPLING MOTION TO LIGHT}

\section{A. Cavity-mediated light-motion coupling}

\section{Model}

The basic setup we use to couple motion to light was originally considered by Zeng and Lin [34. This setup consists of a two-level atom confined in a harmonic trap located inside an optical cavity. The atomic transition of frequency $\omega_{0}$ is coupled to a single mode of the cavity field of frequency $\omega_{\text {cav }}$ and is also driven by an external (classical) laser field of frequency $\omega_{\mathrm{A}}$. The physical setup and excitation scheme are depicted in Fig. 3. The cavity is aligned along the $x$-axis, while the laser field is incident from a direction along the $y$-axis (i.e., perpendicular to the $x$-axis).

The Hamiltonian describing the atom-cavity system, including the atomic motion, takes the form (in a frame rotating at the laser frequency, $\omega_{\mathrm{A}}$ )

$$
\begin{aligned}
\hat{H}_{\mathrm{ac}}= & \sum_{j=} x_{y, z} \hbar \nu_{j}\left(\hat{b}_{j}^{\dagger} \hat{b}_{j}+1 / 2\right)+\hbar \delta_{\mathrm{cA}} \hat{a}^{\dagger} \hat{a}+\hbar \Delta_{0 \mathrm{~A}} \hat{\sigma}_{+} \hat{\sigma}_{-} \\
& +\hbar\left[E_{\mathrm{A}}(\hat{y}, t) \hat{\sigma}_{+}+E_{\mathrm{A}}^{*}(\hat{y}, t) \hat{\sigma}_{-}\right] \\
& +\hbar g_{0} \sin (k \hat{x})\left(\hat{a}^{\dagger} \hat{\sigma}_{-}+\hat{\sigma}_{+} \hat{a}\right) \\
& +\hat{a}^{\dagger} \hat{\Upsilon}_{\mathrm{c}}+\hat{\Upsilon}_{\mathrm{c}}^{\dagger} \hat{a}+\hat{\sigma}_{+} \hat{\Upsilon}_{\mathrm{a}}+\hat{\Upsilon}_{\mathrm{a}}^{\dagger} \hat{\sigma}_{-}
\end{aligned}
$$

Here, $\left\{\nu_{x}, \nu_{y}, \nu_{z}\right\}$ are the harmonic oscillation frequencies along the principal axes of the trap, $\hat{b}_{j}$ and $\hat{a}$ are annihilation operators for the quantized atomic motion and cavity field, respectively, $\hat{\sigma}_{-}=|g\rangle\langle e|$ is the atomic lowering operator, and $\delta_{\mathrm{cA}}=\omega_{\mathrm{cav}}-\omega_{\mathrm{A}}$ and $\Delta_{0 \mathrm{~A}}=\omega_{0}-\omega_{\mathrm{A}}$. The quantity $E_{\mathrm{A}}(\hat{y}, t)$ is the (possibly time-dependent) amplitude of laser field $\mathrm{A}$. The single-photon atom-cavity dipole coupling strength is given by $g_{0}$, while the sine function describes the standing wave structure of the cavity field - we assume that the centre of the trap is located at a node of the cavity field. Finally, the last two terms in (28) describe the couplings of the cavity field mode and the atomic transition to "reservoirs" of external field modes (with $\hat{\Upsilon}_{\mathrm{a}, \mathrm{c}}$ the "reservoir annihilation operators"), which produce damping of the cavity field and (free-space) atomic spontaneous emission, respectively 27. Note that we will neglect any forms of motional decoherence associated with the trap itself.

\section{Elimination of the atomic excited state}

Heisenberg equations of motion are straightforwardly derived from the above Hamiltonian. Assuming the detunings of the light fields from the atomic transition frequency to be very large [i.e., $\Delta_{0 \mathrm{~A}} \gg\left|E_{\mathrm{A}}\right|, g_{0}, \delta, \nu_{j}$ ], atomic spontaneous emission can be neglected and the internal atomic dynamics can be adiabatically eliminated. In the equations of motion, this is done by making the replacement

$$
\hat{\sigma}_{-} \simeq-\frac{1}{\Delta_{0 \mathrm{~A}}}\left[E_{\mathrm{A}}(\hat{y}, t)+g_{0} \sin (k \hat{x}) \hat{a}\right]
$$

in the equations describing the cavity and motional degrees of freedom. The corresponding Hamiltonian then takes the form

$$
\begin{aligned}
\hat{H}_{\mathrm{ac}}= & \sum_{j=x, y, z} \hbar \nu_{j}\left(\hat{b}_{j}^{\dagger} \hat{b}_{j}+1 / 2\right)+\hbar \delta_{\mathrm{cA}} \hat{a}^{\dagger} \hat{a} \\
& -\frac{\hbar\left|E_{\mathrm{A}}(\hat{y}, t)\right|^{2}}{\Delta_{0 \mathrm{~A}}}-\frac{\hbar g_{0}^{2}}{\Delta_{0 \mathrm{~A}}} \sin ^{2}(k \hat{x}) \hat{a}^{\dagger} \hat{a} \\
& -\frac{\hbar g_{0}}{\Delta_{0 \mathrm{~A}}} \sin (k \hat{x})\left[E_{\mathrm{A}}(\hat{y}, t) \hat{a}^{\dagger}+E_{\mathrm{A}}^{*}(\hat{y}, t) \hat{a}\right] \\
& +\hat{a}^{\dagger} \hat{\Upsilon}_{\mathrm{c}}+\hat{\Upsilon}_{\mathrm{c}}^{\dagger} \hat{a} .
\end{aligned}
$$

\section{Lamb-Dicke and rotating-wave approximations}

The size of the harmonic trap is assumed to be small compared to the optical wavelength (Lamb-Dicke approximation); this enables the approximations $\sin (k \hat{x}) \simeq$ $\eta_{x}\left(\hat{b}_{x}+\hat{b}_{x}^{\dagger}\right)$, and $E_{\mathrm{A}}(\hat{y}, t) \simeq \mathcal{E}_{\mathrm{A}}(t) \mathrm{e}^{-i \phi_{\mathrm{A}}}\left[\right.$ with $\mathcal{E}_{\mathrm{A}}(t)$ real $]$. This second approximation would follow, for example, if the laser field forms a standing wave with the trap centered at an antinode, i.e., with $E_{\mathrm{A}}(\hat{y}) \propto \cos (k \hat{y}) \simeq 1$, for $\eta_{y} \ll 1$ (for further discussion of this approximation, see Appendix B).

To second order in $\eta_{x}$, quantum Langevin equations for the field and $x$-dimension motional modes are then

$$
\begin{aligned}
\dot{\hat{a}}= & -\left(\kappa+i \delta_{\mathrm{cA}}\right) \hat{a}+i \frac{\eta_{x}^{2} g_{0}^{2}}{\Delta_{0 \mathrm{~A}}}\left(\hat{b}_{x}+\hat{b}_{x}^{\dagger}\right)^{2} \hat{a} \\
& -i \Omega(t) e^{-i \phi_{\mathrm{A}}}\left(\hat{b}_{x}+\hat{b}_{x}^{\dagger}\right)-\sqrt{2 \kappa} e^{-i \delta_{\mathrm{cA}} t} \hat{a}_{\mathrm{in}}(t), \\
\dot{\hat{b}}_{x}= & -i \nu_{x} \hat{b}_{x}+i \frac{2 \eta_{x}^{2} g_{0}^{2}}{\Delta_{0 \mathrm{~A}}}\left(\hat{b}_{x}+\hat{b}_{x}^{\dagger}\right) \hat{a}^{\dagger} \hat{a} \\
& -i \Omega(t)\left(\hat{a}^{\dagger} e^{-i \phi_{\mathrm{A}}}+\hat{a} e^{i \phi_{\mathrm{A}}}\right)
\end{aligned}
$$

where we have defined

$$
\Omega(t)=-\frac{\eta_{x} g_{0} \mathcal{E}_{\mathrm{A}}(t)}{\Delta_{0 \mathrm{~A}}},
$$

while the operator $\hat{a}_{\text {in }}(t)$ obeys the commutation relation $\left[\hat{a}_{\text {in }}(t), \hat{a}_{\text {in }}^{\dagger}\left(t^{\prime}\right)\right]=\delta\left(t-t^{\prime}\right)$ and describes the quantum noise 
input to the cavity field from the external field (in a frame rotating at the cavity frequency). The parameter $\kappa$ is the cavity field decay rate.

Next, we choose the detuning between the cavity and laser fields to be $\delta_{\mathrm{cA}}=\omega_{\text {cav }}-\omega_{\mathrm{A}}=\nu_{x}$. In the above equations this results in "resonant" and "non-resonant" terms. With the assumption that the trap frequency $\nu_{x}$ is large (which is consistent with the Lamb-Dicke assumption, since $\eta_{x} \propto \nu_{x}^{-1 / 2}$ ), such that $\nu_{x} \gg \kappa,|\Omega(t)|$, the non-resonant, or counter-rotating terms can be neglected in a rotating-wave approximation. This leads to the pair of equations

$$
\begin{array}{r}
\dot{\hat{a}}=-\left(\kappa+i \nu_{x}\right) \hat{a}+i \frac{2 \eta_{x}^{2} g_{0}^{2}}{\Delta_{0 \mathrm{~A}}}\left(\hat{b}_{x}^{\dagger} \hat{b}_{x}+\frac{1}{2}\right) \hat{a} \\
-i \Omega(t) e^{-i \phi_{\mathrm{A}}} \hat{b}_{x}-\sqrt{2 \kappa} e^{-i \nu_{x} t} \hat{a}_{\mathrm{in}}(t), \\
\dot{\hat{b}}_{x}=-i \nu_{x} \hat{b}_{x}+i \frac{2 \eta_{x}^{2} g_{0}^{2}}{\Delta_{0 \mathrm{~A}}} \hat{a}^{\dagger} \hat{a} \hat{b}_{x}-i \Omega(t) e^{i \phi_{\mathrm{A}}} \hat{a} .
\end{array}
$$

The terms of second order in $\eta_{x}$ describe phonon- or photon-number-dependent phase shifts; these will in general be very small and can be neglected (although they are retained for numerical calculations) 35], which means that the effective interaction between the cavity and motional modes is simply a linear coupling of the form

$$
\hat{H}_{\mathrm{eff}}=\hbar \Omega(t)\left(\hat{a}^{\dagger} \hat{b}_{x} e^{-i \phi_{\mathrm{A}}}+\hat{b}_{x}^{\dagger} \hat{a} e^{i \phi_{\mathrm{A}}}\right) .
$$

\section{Adiabatic elimination of the cavity mode}

Although not essential for our purposes, a further simplification of the dynamics is possible if the decay rate $\kappa$ of the cavity field is sufficiently large that the cavity mode dynamics can also be adiabatically eliminated. In particular, if $\kappa \gg|\Omega(t)|$ (but still with $\nu_{x} \gg \kappa$ ), then the equation for the motional mode reduces to

$$
\dot{\hat{b}}_{x} \simeq-\left[\Gamma(t)+i \nu_{x}\right] \hat{b}_{x}+e^{i \phi_{\mathrm{A}}} \sqrt{2 \Gamma(t)} e^{-i \nu_{x} t} \hat{a}_{\mathrm{in}}(t),
$$

where we define

$$
\Gamma(t)=\Omega(t)^{2} / \kappa
$$

The motional dynamics thus reduces to that of a simple damped harmonic oscillator, with the (possibly) timedependent damping rate $\Gamma(t)$. However, the quantum noise operator appearing in (37) corresponds to the light field incident upon the cavity, and hence one realizes a simple coupling of the motional mode to propagating light fields external to the cavity. More precisely, from the input-output theory of optical cavities [27,36], it can be shown that the cavity output field is given, under the present circumstances, by

$$
\hat{a}_{\mathrm{out}}(t) \simeq-\hat{a}_{\mathrm{in}}(t)-\sqrt{2 \Gamma(t)} \tilde{b}_{x}(t) .
$$

where $\tilde{b}_{x}=\mathrm{e}^{i \nu_{x} t} \hat{b}_{x}$, and we have set $\phi_{\mathrm{A}}=0$ for simplicity. So, given a vacuum input field to the cavity, the output light field is determined by the motional state of the atom confined inside the cavity. In this way, nonclassical motional states can be converted into nonclassical light fields; for example, entanglement between the $x$ dimension motional mode and, say, the $z$-dimension motional mode can be converted into entanglement between the $z$-dimension motional mode and the propagating output light field. This light field may then be coupled to another atom-cavity system to generate distributed motional state entanglement.

\section{B. Motional state transfer between distant locations}

Following work by Cirac et al. 37] on the transmission of a qubit between two nodes of a quantum network, it is shown in 12] that if the output field from one of our atom-cavity configurations is incident on a second such atom-cavity configuration, with the coupling between systems being unidirectional, then with suitably tailored laser pulses $\mathcal{E}_{\mathrm{A} 1}(t)$ and $\mathcal{E}_{\mathrm{A} 2}(t)$ applied at the two sites (amounting essentially to impedance matching, such that all of the light exiting the first cavity is absorbed by the second cavity) one may realize the motional state transfer

$$
|\phi\rangle_{x}^{(1)} \otimes|0\rangle_{x}^{(2)} \rightarrow|0\rangle_{x}^{(1)} \otimes|\phi\rangle_{x}^{(2)},
$$

where $|\phi\rangle_{x}$ is an arbitrary quantum state describing the motion along the $x$-axis. Note that the cavity fields begin and end the transfer in the vacuum state.

The scheme is able to operate in the regime where $\Omega(t)$ and $\kappa$ are comparable (in which case the transfer rate is essentially determined by $\kappa$ ), but again the analysis simplifies in the case where the cavity modes can be adiabatically eliminated as above. In this case, a master equation for the reduced density matrix describing the motional states of the two atoms, $\tilde{\rho}_{x}$, can be derived (in the rotating frame) as 12 ]

$$
\begin{aligned}
\dot{\tilde{\rho}}_{x}= & \Gamma_{1}(t)\left(2 \tilde{b}_{1 x} \tilde{\rho}_{x} \tilde{b}_{1 x}^{\dagger}-\tilde{b}_{1 x}^{\dagger} \tilde{b}_{1 x} \tilde{\rho}_{x}-\tilde{\rho}_{x} \tilde{b}_{1 x}^{\dagger} \tilde{b}_{1 x}\right) \\
& +\Gamma_{2}(t)\left(2 \tilde{b}_{2 x} \tilde{\rho}_{x} \tilde{b}_{2 x}^{\dagger}-\tilde{b}_{2 x}^{\dagger} \tilde{b}_{2 x} \tilde{\rho}_{x}-\tilde{\rho}_{x} \tilde{b}_{2 x}^{\dagger} \tilde{b}_{2 x}\right) \\
& +2 \sqrt{\Gamma_{1}(t) \Gamma_{2}(t)}\left\{\left[\tilde{b}_{2 x}^{\dagger}, \tilde{b}_{1 x} \tilde{\rho}_{x}\right] e^{-i\left(\phi_{1 \mathrm{~A}}-\phi_{2 \mathrm{~A}}\right)}\right. \\
& \left.+\left[\tilde{\rho}_{x} \tilde{b}_{1 x}^{\dagger}, \tilde{b}_{2 x}\right] e^{i\left(\phi_{\mathrm{A} 1}-\phi_{\mathrm{A} 2}\right)}\right\}
\end{aligned}
$$

Example pulse shapes for this regime, specified through the effective coupling rates of the motional modes to the external light fields, $\Gamma_{1}(t)$ and $\Gamma_{2}(t)$, are $\left(\right.$ taking $\left.\phi_{\mathrm{A} 1}=\phi_{\mathrm{A} 2}\right)$

$$
\Gamma_{1}(t)=\Gamma \frac{\mathrm{e}^{\Gamma t}}{\mathrm{e}^{\Gamma t}+\mathrm{e}^{-\Gamma t}}, \quad \Gamma_{2}(t)=\Gamma_{1}(-t),
$$


assuming the transfer starts at $t=-\infty$ and concludes at $t=+\infty$, with $\Gamma$ a constant. Armed with this capability, we are able to distribute quantum states of a material oscillator, and generate entanglement, between macroscopically-separated locations.

\section{Numerical analysis}

\section{Damped harmonic oscillator model}

The description of the motional mode dynamics in terms of a linearly-damped harmonic oscillator coupled to propagating light fields, Eq.(37), represents a tremendous simplification of the model and offers a very direct and transparent scheme for state transfer between motion and light. It is important then to gauge the validity of this simplification; here we present some results from a numerical analysis of the model starting from the Hamiltonian

$$
\begin{aligned}
\hat{H}_{\mathrm{ac}}= & \hbar \nu_{x}\left(\hat{b}_{x}^{\dagger} \hat{b}_{x}+1 / 2\right)+\hbar \delta_{\mathrm{cA}} \hat{a}^{\dagger} \hat{a} \\
& -\frac{\hbar \mathcal{E}_{\mathrm{A}}^{2}}{\Delta_{0 \mathrm{~A}}}-\frac{\hbar g_{0}^{2}}{\Delta_{0 \mathrm{~A}}} \sin ^{2}(k \hat{x}) \hat{a}^{\dagger} \hat{a} \\
& -\frac{\hbar g_{0} \mathcal{E}_{\mathrm{A}}}{\Delta_{0 \mathrm{~A}}} \sin (k \hat{x})\left(e^{-i \phi_{\mathrm{A}}} \hat{a}^{\dagger}+e^{i \phi_{\mathrm{A}}} \hat{a}\right),
\end{aligned}
$$

and master equation

$$
\dot{\hat{\rho}}=-\frac{i}{\hbar}\left[\hat{H}_{\mathrm{ac}}, \hat{\rho}\right]+\kappa\left(2 \hat{a} \hat{\rho} \hat{a}^{\dagger}-\hat{a}^{\dagger} \hat{a} \hat{\rho}-\hat{\rho} \hat{a}^{\dagger} \hat{a}\right)
$$

where the last term in (44) describes cavity damping. The Hamiltonian (43) is the one-dimensional form of 30 and neglects the position dependence of $\mathcal{E}_{\mathrm{A}}$ (based on earlier arguments; see Appendix B). These equations make no assumption about the Lamb-Dicke parameter in the $x$ direction and retain the dynamics of the cavity mode.

We solve the master equation (44) numerically using truncated number state bases for the (harmonic oscillator) cavity and motional modes. The particular example we have concentrated on is the decay of an initial coherent state of the motional mode, since this allows a simple analytical solution in the ideal case where the motional mode dynamics is exactly described by (37). In particular, for an initial coherent amplitude $\alpha$, the state evolves as $|\psi(t)\rangle_{x}=e^{-i \nu_{x} t / 2}\left|\alpha e^{-\left(i \nu_{x}+\Gamma\right) t}\right\rangle_{x}$ in the ideal case.

A natural quantity to consider then in comparing the simulated evolution with the ideal behavior is the fidelity

$$
f(t)={ }_{x}\left\langle\alpha e^{-\left(i \nu_{x}+\Gamma\right) t}\left|\hat{\rho}_{x}\right| \alpha e^{-\left(i \nu_{x}+\Gamma\right) t}\right\rangle_{x},
$$

where $\hat{\rho}_{x}=\operatorname{Tr}_{\text {cav }}\{\hat{\rho}\}$ is the reduced density operator for the motional mode and

$$
\Gamma=\frac{1}{\kappa}\left(\frac{\eta_{x} g_{o} \mathcal{E}_{\mathrm{A}}}{\Delta_{0 \mathrm{~A}}}\right)^{2} .
$$

a. Results Results for two values of the Lamb-Dicke parameter, $\eta_{x}=0.1$ and $\eta_{x}=0.15$, are shown in Fig. 4, where we plot the magnitudes of the motional mode and cavity mode amplitudes as a function of time, with the coherent state amplitude chosen to be $\alpha=\sqrt{10}$. In dimensionless units we choose $\kappa=1, \nu_{x}=\delta_{\mathrm{cA}}=10$, $g_{0}^{2} / \Delta_{0 \mathrm{~A}}=0.2$, and $\eta_{x} g_{0} \mathcal{E}_{\mathrm{A}} / \Delta_{0 \mathrm{~A}}=0.1(\ll \kappa)$, corresponding to $\Gamma=0.01$. In Fig. $4(\mathrm{a}),\left|\left\langle\hat{b}_{x}(t)\right\rangle\right|$ is seen to follow very closely the ideal behavior of a decaying coherent state, while Figs. 4(b,c) demonstrate that the cavity mode, after an initial transient period, adiabatically follows the motional mode. There is a small but noticeable improvement (with respect to the ideal behavior) with the decrease in $\eta_{x}$ from 0.15 to 0.1 . Further improvements occur with smaller $\left(\eta_{x} g_{0} \mathcal{E}_{\mathrm{A}} / \Delta_{0 \mathrm{~A}}\right) / \kappa$ (condition of adiabaticity) and larger $\nu_{x} / \kappa$ (rotating-wave approximation with respect to the trap frequency), as one would expect.

The fidelity $f(t)$ is plotted in Fig. 5, from which it is clear that, for $\eta=0.1$, the motional state remains close to the desired state at all times (at large $t$, of course, the states all approach the ground state). For $\eta_{x}=0.15$ the deviation is more significant and the mapping of the motional state onto the light field is evidently degraded.

b. Lamb-Dicke approximation Let us return briefly to some of the approximations made in deriving the ideal model. If we consider the Hamiltonian (43), the term proportional to $g_{0}^{2} / \Delta_{0 \mathrm{~A}}$ is essentially negligible under the present circumstances due to the smallness of $\eta_{x}$ and of the intracavity photon number $\left(\left\langle\hat{a}^{\dagger} \hat{a}\right\rangle<0.1\right.$ at early times and decreases with time). Consider now the expansion of $\sin (k \hat{x})$. To third order in $\eta_{x}$

$$
\begin{aligned}
\sin (k \hat{x}) & =\eta_{x}\left(\hat{b}_{x}+\hat{b}_{x}^{\dagger}\right)-\frac{\eta_{x}^{3}}{3 !}\left(\hat{b}_{x}+\hat{b}_{x}^{\dagger}\right)^{3} \\
& =\eta_{x}\left(1-\frac{\eta_{x}^{2}}{2}-\frac{\eta_{x}^{2}}{2} \hat{b}_{x}^{\dagger} \hat{b}_{x}-\frac{\eta_{x}^{2}}{6} \hat{b}_{x}^{2}\right) \hat{b}_{x}+\text { h.c.. }
\end{aligned}
$$

The reduction of the atom-cavity dynamics to a coupling of the form $\hat{a}^{\dagger} \hat{b}_{x}+$ h.c. requires that the terms in the brackets proportional to $\eta_{x}^{2}$ have negligible effect (compared to 1$)$. The combination of small $\eta_{x}$ and rapid rotation means that the terms $\eta_{x}^{2} \hat{b}_{x}^{2} / 6$ and $\eta_{x}^{2} \hat{b}_{x}^{\dagger 2} / 6$ should be negligible, leaving a condition of the form

$$
\frac{1}{2} \eta_{x}^{2}\left(1+\bar{n}_{x}\right) \ll 1,
$$

where $\bar{n}_{x} \equiv\left\langle\hat{b}_{x}^{\dagger} \hat{b}_{x}\right\rangle$. This condition is reasonably well satisfied for the numerical examples considered, but a stronger condition would take into account the width of the number state distribution, i.e., the fact that vibrational population can reside in appreciable amounts in number states $|n\rangle_{x}$ such that $n>\bar{n}_{x}$. If $\sigma_{\bar{n}_{x}}^{2}$ is the variance of the number state distribution, then such a condition might take the form

$$
\frac{1}{2} \eta_{x}^{2}\left(1+\bar{n}_{x}+a \sigma_{\bar{n}_{x}}\right) \ll 1
$$


where $a \sim 2-3$ (i.e., several standard deviations from the mean). For the coherent state considered above, $\sigma_{\bar{n}_{x}}=$ $\sqrt{\bar{n}_{x}}=\sqrt{10}$, leading to a condition (taking $a=3$ )

$$
\frac{1}{2} \eta_{x}^{2}\left(1+\bar{n}_{x}+3 \sigma_{\bar{n}_{x}}\right) \simeq 10 \eta_{x}^{2} \ll 1 .
$$

With $\eta_{x}=0.15,10 \eta_{x}^{2}=0.225$, and so one might expect that the Lamb-Dicke approximation starts to break down.

In the case of a two-mode squeezed state of the motion, the number state distribution of the individual modes is akin to that of a thermal mode, for which $\sigma_{\bar{n}_{x}}=\left(\bar{n}_{x}^{2}+\right.$ $\left.\bar{n}_{x}\right)^{1 / 2} \simeq \bar{n}_{x}+1 / 2$ for $\bar{n}_{x}>1$. With $a=3$, we then require that

$$
\frac{1}{2} \eta_{x}^{2}\left(4 \bar{n}_{x}+5 / 2\right) \ll 1
$$

which, for $\eta_{x}=0.1$, reduces to $\bar{n}_{x}=\sinh ^{2}(r) \ll 50$. This condition is reasonably well satisfied for values of $r$ up to $\sim 1.6$; beyond this, a smaller value of $\eta_{x}$ would be desirable.

\section{Motional state transfer}

The next problem we wish to examine in more detail is that of transferring a motional quantum state from one atom to another at a distant site using the procedure outlined in Section III.B and depicted schematically in Fig. 6. The coupling laser fields, and hence the equations of motion, now have an explicit time dependence. This, combined with the increased dimensionality of the problem (now that we have two atom-cavity systems to simulate), leads us to employ the technique of MonteCarlo wave function simulation (see, for example, [36]).

In fact, the Monte-Carlo wave function technique is not only convenient, but also highly appropriate for modeling and analyzing the state transfer procedure as shown in Fig. 6. In this approach, the evolution of our quantum system is simulated by propagating a wave function $|\Psi(t)\rangle$ according to the Schrödinger equation

$$
\frac{\partial}{\partial t}|\Psi(t)\rangle=-\frac{i}{\hbar} \hat{H}_{\mathrm{eff}}(t)|\Psi(t)\rangle,
$$

where $\hat{H}_{\text {eff }}(t)$ is a non-Hermitian effective Hamiltonian. For the state-transfer configuration we are considering here, our effective Hamiltonian takes the form

$$
\begin{aligned}
\hat{H}_{\mathrm{eff}}(t)= & \hat{H}_{\mathrm{ac}}^{(1)}(t)+\hat{H}_{\mathrm{ac}}^{(2)}(t) \\
& -i \hbar \kappa_{1} \hat{a}_{1}^{\dagger} \hat{a}_{1}-i \hbar \kappa_{2} \hat{a}_{2}^{\dagger} \hat{a}_{2}-2 i \hbar \sqrt{\kappa_{1} \kappa_{2}} \hat{a}_{2}^{\dagger} \hat{a}_{1},
\end{aligned}
$$

with

$$
\begin{aligned}
\hat{H}_{\mathrm{ac}}^{(j)}(t) & =\hbar \nu_{x j} \hat{b}_{x j}^{\dagger} \hat{b}_{x j}+\hbar \delta_{\mathrm{cA}}^{(j)} \hat{a}_{j}^{\dagger} \hat{a}_{j} \\
& -\frac{\hbar g_{0 j}^{2}}{\Delta_{0 \mathrm{~A}}} \sin ^{2}\left(k \hat{x}_{j}\right) \hat{a}_{j}^{\dagger} \hat{a}_{j} \\
& -\frac{\hbar g_{0 j} \mathcal{E}_{\mathrm{A} j}(t)}{\Delta_{0 \mathrm{~A}}} \sin \left(k \hat{x}_{j}\right)\left(e^{-i \phi_{\mathrm{A} j}} \hat{a}_{j}^{\dagger}+e^{i \phi_{\mathrm{A} j}} \hat{a}_{j}\right) .
\end{aligned}
$$

[For simplicity, we now omit constant energy shifts from the Hamiltonians $\hat{H}_{\mathrm{ac}}^{(j)}(t)$, and the frequencies of the two coupling lasers are assumed to be the same $\left(\omega_{\mathrm{A}}\right)$.] The second line in (53) follows from the cascaded-systems formalism in which one assumes a unidirectional coupling between the cavities 36, 38, 39.

The propagation described by (52) is interrupted at random times $\left\{t_{r}\right\}$ by wave function collapses, or quantum jumps,

$$
\left|\Psi\left(t_{r}+d t\right)\right\rangle=\frac{\hat{C}\left|\Psi\left(t_{r}\right)\right\rangle}{\left\langle\Psi\left(t_{r}\right)\left|\hat{C}^{\dagger} \hat{C}\right| \Psi\left(t_{r}\right)\right\rangle^{1 / 2}}
$$

(which include renormalization of the wave function), where $\hat{C}$ is an appropriate jump operator and the probability density for a jump to occur during the time interval from $t$ to $t+d t$ is $\left\langle\Psi(t)\left|\hat{C}^{\dagger} \hat{C}\right| \Psi(t)\right\rangle d t$. For our situation the jump operator is

$$
\hat{C}=\sqrt{\kappa_{1}} \hat{a}_{1}+\sqrt{\kappa_{2}} \hat{a}_{2},
$$

which can be identified with the destructive detection of a photon by the photodetector monitoring the output channel from the second cavity.

For ideal quantum transmission we require that a quantum jump (that is, a photon detection) never occurs, i.e., $\hat{C}|\Psi(t)\rangle=0$ for all times $t$ (which also means that the norm of the wave function remains equal to 1 at all times). In other words, all of the quantum information is transferred from atom 1 to atom 2 and none is lost to light fields propagating away from the system. The laser pulse profiles derived from the simplified master equation model (41) should approximately satisfy this condition and facilitate high-fidelity state transfer, provided the various parameters of the system are chosen appropriately. Here we want to assess more carefully the performance of these profiles for a more comprehensive model of the atom-cavity dynamics, as described by the Hamiltonians in (54).

We assume an initial state of the form

$$
|\Psi(t=-\infty)\rangle=|\phi\rangle_{x}^{(1)} \otimes|0\rangle_{\mathrm{cav}}^{(1)} \otimes|0\rangle_{\mathrm{cav}}^{(2)} \otimes|0\rangle_{x}^{(2)},
$$

where $|\phi\rangle_{x}^{(1)}$ is the particular motional quantum state to be transmitted. The target state of the transmission is then

$$
|\Psi(t=+\infty)\rangle_{\text {target }}=|0\rangle_{x}^{(1)} \otimes|0\rangle_{\text {cav }}^{(1)} \otimes|0\rangle_{\text {cav }}^{(2)} \otimes|\phi\rangle_{x}^{(2)} .
$$

For simplicity, we will also assume identical atom-cavity systems, i.e.,

$$
\begin{aligned}
& \nu_{x 1}=\nu_{x 2} \equiv \nu_{x}, \quad \eta_{x 1}=\eta_{x 2} \equiv \eta_{x} \\
& g_{01}=g_{02} \equiv g_{0}, \quad \kappa_{1}=\kappa_{2} \equiv \kappa, \quad \delta_{\mathrm{cA}}^{(1)}=\delta_{\mathrm{cA}}^{(2)} \equiv \delta_{\mathrm{cA}} .
\end{aligned}
$$


a. Laser pulse profiles The time dependence of the laser field $\mathcal{E}_{\mathrm{A} 1}(t)$ is chosen to satisfy

$$
\frac{1}{\kappa}\left[\frac{\eta_{x} g_{0} \mathcal{E}_{\mathrm{A} 1}(t)}{\Delta_{0 \mathrm{~A}}}\right]^{2} \equiv \Gamma_{1}(t)=\Gamma \frac{e^{\Gamma t}}{e^{\Gamma t}+e^{-\Gamma t}},
$$

with

$$
\Gamma=\frac{1}{\kappa}\left[\frac{\eta_{x} g_{0} \mathcal{E}_{\mathrm{A} 1}^{\max }}{\Delta_{0 \mathrm{~A}}}\right]^{2},
$$

while $\mathcal{E}_{\mathrm{A} 2}(t)$ is chosen such that $\Gamma_{2}(t)=\Gamma_{1}(-t)$. Note again that these example forms for the temporal profiles of the laser fields are derived from a theoretical analysis in which the dynamics of the cavity modes are adiabatically eliminated, and hence, depending on the choices of parameters, they may not be optimal choices. We will return to this point when we present the numerical results.

b. Truncation of trigonometric functions To help speed up our computations we have in fact performed most of our simulations using expansions of the operators $\sin ^{2}\left(k \hat{x}_{j}\right)$ and $\sin \left(k \hat{x}_{j}\right)$ that are truncated to third order in the Lamb-Dicke parameter $\eta_{x}$. For the range of parameters considered here, we find little difference between results obtained using the full trigonometric forms and those using the truncated versions. With the truncated versions it is also straightforward to move to a rotating frame so as to remove the systematic (fast) evolution given by the first line in (54). This does of course result in rapidly rotating factors multiplying some of the remaining terms of the Hamiltonians [in particular, factors of the forms $e^{ \pm i\left(\nu_{x}+\delta_{\mathrm{cA}}\right) t}$ or $\left.e^{ \pm i\left(3 \nu_{x} \pm \delta_{\mathrm{cA}}\right) t}\right]$, and we retain these terms in the simulations.

c. Fidelity of the transmission In the context of our wave function simulations, the ideal situation corresponds to the case where, following the laser pulse sequence, the norm of the wave function is still equal to 1 , and hence no jumps have occurred. However, due to non-ideal operating conditions (for example, rapidly rotating "off-resonant" terms making a finite contribution to the dynamics) the norm of the wave function does decay and hence there is a finite possibility of a photon detection and consequent loss of information. Now, in a typical application of the Monte-Carlo wave function approach one averages over many trajectories to obtain a density operator for the system. From this density operator one could, in the present context, compute the average fidelity for the quantum state transmission.

However, the decay of the norm in a single trajectory already provides us with a good indicator of the performance of the transmission. If, for example, during the pulse sequence and state transfer the norm decays to a value of 0.9 (without any jumps occurring), then we can make the general statement that in $90 \%$ of our attempts the state will be transferred successfully. Note that, in the "no-jump" case, on renormalizing the simulated wave function $\left|\Psi\left(t_{\mathrm{f}}\right)\right\rangle_{\operatorname{sim}}$ (where $t_{\mathrm{f}}$ is the finishing time for the simulation) we find that the fidelity of the transmission, defined to be

$$
F=\left|\operatorname{sim}\left\langle\Psi\left(t_{\mathrm{f}}\right) \mid \Psi(+\infty)\right\rangle_{\text {target }}\right|^{2},
$$

is very close to 1 (i.e., $\geq 0.99$ ) for all of the numerical examples we consider below. The value of 0.9 then also essentially sets a lower bound on the average fidelity of the state transmission. In the $10 \%$ of cases where one or more quantum jumps do occur the final state $\left|\Psi\left(t_{\mathrm{f}}\right)\right\rangle_{\text {sim }}$ may, depending on the precise nature of the state being transferred, still have a finite (and even substantial) overlap with the target state, and so the average fidelity will actually be larger than 0.9 .

From a practical point of view, it is also worth noting that it would in principle be possible to post-select high-fidelity state transmissions by monitoring the output from the second cavity. If a photon is not detected in this output then the transmission is known to have been successful (assuming perfect detection efficiency). If a photon is detected then the system can be reset and the transfer attempted again (or some form of error correction procedure could be applied).

$d$. Results As the state to be transferred, we have concentrated on the following examples: (i) a truncated phase state,

$$
|\phi\rangle_{x}^{(1)}=\frac{1}{\sqrt{N+1}} \sum_{n=0}^{N}|n\rangle_{x}^{(1)}
$$

i.e., a uniformly-weighted coherent superposition of the first $N+1$ Fock states, (ii) a pure Fock state

$$
|\phi\rangle_{x}^{(1)}=|N\rangle_{x}^{(1)},
$$

and (iii) a Schrödinger Cat state

$$
|\phi\rangle_{x}^{(1)}=\frac{1}{\mathcal{N}_{+}}\left(|\alpha\rangle_{x}^{(1)}+|-\alpha\rangle_{x}^{(1)}\right),
$$

with $|\alpha\rangle_{x}^{(1)}$ a coherent state and $\mathcal{N}_{+}=\left[2\left(1+e^{-2|\alpha|^{2}}\right)\right]^{1 / 2}$.

In dimensionless units, we again set $\kappa=1$ and choose $g_{0}^{2} / \Delta_{0 \mathrm{~A}}=0.2$ and $\left(g_{0} \mathcal{E}_{\mathrm{A}}^{\max }\right) / \Delta_{0 \mathrm{~A}}=1$. For $\eta_{x}=0.1$ this gives $\left(\eta_{x} g_{0} \mathcal{E}_{\mathrm{A}}^{\max }\right) / \Delta_{0 \mathrm{~A}}=0.1 \kappa$ and so the adiabatic approximation $\left[\left(\eta_{x} g_{0} \mathcal{E}_{\mathrm{A}}^{\max }\right) / \Delta_{0 \mathrm{~A}} \ll \kappa\right]$ used in deriving the laser pulse shapes should be reasonably good. Indeed, we find that the improvement in the fidelity of the transfer one obtains when $\eta_{x}$ is reduced from 0.1 to 0.0707 (see tables below) results primarily from an improvement in the Lamb-Dicke approximation rather than in the adiabatic approximation.

(i) Truncated phase state: Some results illustrating the performance of the transfer for this state are presented in Tables $\amalg(N=10)$ and $\amalg(N=20)$. We have considered various combinations of the trapping frequency $\nu_{x}$ and the Lamb-Dicke parameter $\eta_{x}$. The third column gives the norm of the wave function at the conclusion of the transfer operation (with quantum jumps "turned off"), 
which, as discussed earlier, effectively amounts to a lower bound on the average transfer fidelity, $F_{\text {ave }}$.

As one can see, a large trapping frequency is very important for obtaining a high average transfer fidelity. In particular, a value $\nu_{x} / \kappa \geq 10$ is necessary if one wishes to obtain a success rate exceeding $\sim 80 \%$ for the case $N=20$. As the dimensionality of the motional state being transferred increases (i.e., higher Fock states are populated), the size of $\eta_{x}$ also becomes more critical. This can be seen by comparing the results in Tables II and III. Smaller values of $\eta_{x}$ are clearly required (for a given value of $\nu_{x}$ ) in order for the state $1 / \sqrt{21} \sum_{n=0}^{20}|n\rangle_{x}$ to be transferred with a fidelity comparable to that for the state $1 / \sqrt{21} \sum_{n=0}^{10}|n\rangle_{x}$. The relative improvement in performance with a decrease is $\eta_{x}$ is also more pronounced for the state of higher dimensionality.

With the inclusion of quantum jumps some interesting and complicated behavior is observed in the individual trajectories. For the phase state considered the overlap of the transmitted state with the ideal state can be substantial $(\sim 0.40-0.75)$, but the precise nature of the transmitted state depends crucially on the time at which each jump occurs.

(ii) Fock state: Results for transmission of the Fock state $|n=10\rangle_{x}$ are given in Table IV. The performance is in fact comparable to that for the phase state $1 / \sqrt{21} \sum_{n=0}^{20}|n\rangle_{x}$, which actually has the same mean phonon number of 10 .

The effect of quantum jumps on the transfer is, as one would expect, more severe than in the case of the phase state; overlap with the state $|n=10\rangle_{x}$ disappears completely with a single photon detection. However, after the jump the resulting final state is not necessarily what one might naively expect (i.e., the state $|n=9\rangle_{x}$ ). It is possible, as a result of the finite effects of "non-resonant" terms in the dynamics, for the transferred state to take the form of a superposition of the states $|n=9\rangle_{x}$ and $|n=11\rangle_{x}$.

(iii) Schrödinger Cat state: Finally, we consider the transfer of a mesoscopic superposition of coherent states of the form $\mathcal{N}_{+}^{-1}\left(|\alpha\rangle_{x}+|-\alpha\rangle_{x}\right)$, with $\alpha=\sqrt{10}$. This state also has a mean excitation (phonon) number equal to 10 and the results shown in Table $\mathrm{V}$ are very similar to those of the previous two examples.

The effect of quantum jumps is particularly interesting in this case, as we find that, with a single photon detection, the transferred state is in general very close to the state $\mathcal{N}_{-}^{-1}\left(|\alpha\rangle_{x}-|-\alpha\rangle_{x}\right)$ (i.e., an "odd" Schrödinger Cat state), suggesting that an operation on the motional state amounting to an application of the annihilation operator $\hat{b}_{x 2}$ would largely restore the original state. Note that the even and odd Schrödinger Cat states $\mathcal{N}_{+}^{-1}\left(|\alpha\rangle_{x}+|-\alpha\rangle_{x}\right)$ and $\mathcal{N}_{-}^{-1}\left(|\alpha\rangle_{x}-|-\alpha\rangle_{x}\right)$ have been proposed, in the context of quantum computation, as logical qubit encodings for the correction of bit-flip errors caused by amplitude damping (as occurs in our system) [40]. They have also been proposed for use in a secure quantum key distribution protocol 41].

e. Atomic spontaneous emission While our model and simulations have included cavity damping, we have until now ignored any effects associated with atomic spontaneous emission, which arises from the small but finite probability for the atom to be in its excited internal state $|e\rangle$. In Appendix A (Part 2) an approximate expression is derived for the rate at which atomic spontaneous emission is expected to influence the motional state of the atom. In order for this rate to be much slower than the characteristic state transfer rate $\Gamma$ of the present configuration, one requires that the condition

$$
\frac{10 g_{0}^{2}}{\kappa \gamma} \gg 1
$$

be satisfied, where $\gamma$ is the atomic spontaneous emission rate. Hence, one desires the regime of strong-coupling cavity QED, for which $g_{0}^{2} /(\kappa \gamma) \gtrsim 1$.

f. Experimental prospects Let us focus again on the case of trapped ${ }^{9} \mathrm{Be}^{+}$ions, although other ionic or atomic species could evidently be considered (and might perhaps be preferable from the point of view of cavity QED experiments). The ${ }^{2} \mathrm{~S}_{1 / 2} \leftrightarrow{ }^{2} \mathrm{P}_{1 / 2}$ transition wavelength is $\lambda=313 \mathrm{~nm}$ and the transition linewidth is $\gamma /(2 \pi)=$ 19.4 MHz. If we assume, for example, that the mirrors forming the cavity have radii of curvature equal to $5 \mathrm{~cm}$ and are separated by a distance $l=1(2) \mathrm{mm}$, then $g_{0} /(2 \pi)=5.3(3.1) \mathrm{MHz}$. For a cavity finesse of 75,000 one obtains $\kappa /(2 \pi)=1.0(0.5) \mathrm{MHz}$, and so $10 g_{0}^{2} /(\kappa \gamma)=$ 14 (10), while a trap frequency of $\nu_{x} /(2 \pi)=22 \mathrm{MHz}$ (corresponding to a Lamb-Dicke parameter $\eta_{x}=0.1$ ) gives $\nu_{x} / \kappa=22(44)$. With these parameters one would anticipate a state transfer rate $\Gamma /(2 \pi) \sim 10-20 \mathrm{kHz}$. Note that the timescales for motional decoherence and heating observed in recent trapped ion experiments are of the order of milliseconds or longer [17, 18, 42 .

There are of course many other possible combinations of parameters which should satisfy (at least approximately) the essential requirements of the scheme. Much larger cavity finesses may be possible, but challenging, at this wavelength, allowing smaller mirror separation and larger dipole coupling strength $g_{0}$ (which increases as the cavity mode volume decreases). However, it may be difficult in practice to bring mirrors very close together about an ion trap (for example, because of the technical problem of charge build-up on the mirrors). For this reason, it is perhaps advantageous to consider neutral atom experiments (in particular, alkali atom experiments), where, in fact, optical dipole traps (see, for example, 43]) or microscopic magnetic traps (see, for example, (44) should allow confinement of atoms in the Lamb-Dicke regime with trapping frequencies also in the $\mathrm{MHz}$ range. Furthermore, cavity QED experiments with alkali atoms are already highly developed (for recent experiments, see [43,45,46]) and spectacular cavity finesses can be achieved at the relevant (longer) wavelengths [47]. 


\section{Multiple cavity-confined atoms}

It is interesting to consider the possibility of having more than one atom coupled to the cavity field at a time. The kind of situation one might imagine is like that realized in the recent experiment of Ye et al. [43], in which (single) atoms were trapped inside a microscopic optical cavity using a far-off-resonance dipole-force trap (FORT). The FORT was actually produced by excitation of an "auxiliary" longitudinal cavity mode, and so had a standing-wave structure colinear with (but of slightly different periodicity to) the cavity QED mode of interest. Hence, given sufficiently strong confinement, the FORT can be regarded as a chain of individual cavity-confined microtraps, each of which could in principle be occupied by a different atom.

With, for simplicity, a single coupling field $\mathcal{E}_{\mathrm{A}}$ incident on the atoms from the side of the cavity, a Hamiltonian for the system can be written as (assuming a single microtrap frequency $\nu_{x}$, and tight confinement along transverse directions as well)

$$
\begin{aligned}
\hat{H}_{\mathrm{ac}} & =\sum_{j} \hbar \nu_{x}\left(\hat{b}_{x j}^{\dagger} \hat{b}_{x j}+1 / 2\right)+\hbar \delta_{\mathrm{cA}} \hat{a}^{\dagger} \hat{a} \\
& -\sum_{j} \frac{\hbar g_{0}^{2}}{\Delta_{0 \mathrm{~A}}} \sin ^{2}\left(k \hat{x}_{j}+\theta_{j}\right) \hat{a}^{\dagger} \hat{a}-\sum_{j} \frac{\hbar \mathcal{E}_{\mathrm{A}}^{2}}{\Delta_{0 \mathrm{~A}}} \\
& -\sum_{j} \frac{\hbar g_{0} \mathcal{E}_{\mathrm{A}}}{\Delta_{0 \mathrm{~A}}} \sin \left(k \hat{x}_{j}+\theta_{j}\right)\left(e^{-i \phi_{\mathrm{A}}} \hat{a}^{\dagger}+e^{i \phi_{\mathrm{A}}} \hat{a}\right),
\end{aligned}
$$

where $\theta_{j}$ defines the position of the center of the $j$-th microtrap relative to a node of the cavity QED field.

In the Lamb-Dicke limit we can write

$$
\begin{aligned}
\sin \left(k \hat{x}_{j}+\theta_{j}\right) & =\sin \left(k \hat{x}_{j}\right) \cos \left(\theta_{j}\right)+\cos \left(k \hat{x}_{j}\right) \sin \left(\theta_{j}\right) \\
& \simeq \eta_{x}\left(\hat{b}_{x j}+\hat{b}_{x j}^{\dagger}\right) \cos \left(\theta_{j}\right)+\sin \left(\theta_{j}\right) .
\end{aligned}
$$

Assuming that $g_{0}^{2} / \Delta_{0 \mathrm{~A}} \ll \delta=\nu_{x}$ and that $g_{0}\left\langle\hat{a}^{\dagger} \hat{a}\right\rangle^{1 / 2} \ll$ $\mathcal{E}_{\mathrm{A}}$, we can neglect the term in (67) proportional to $g_{0}^{2} / \Delta_{0 \mathrm{~A}}$, while in the last term we can neglect the contribution proportional to $\sin \left(\theta_{j}\right)\left(e^{-i \phi_{\mathrm{A}}} \hat{a}^{\dagger}+e^{i \phi_{\mathrm{A}}} \hat{a}\right)$ as this term is rapidly rotating (at frequencies $\pm \delta_{\mathrm{cA}}$, with $\delta_{\mathrm{cA}}=$ $\left.\nu_{x} \gg \kappa, g_{0} \mathcal{E}_{\mathrm{A}} / \Delta_{0 \mathrm{~A}}\right)$ compared to terms of the form $\hat{a}^{\dagger} \hat{b}_{x j}$ and $\hat{b}_{x j}^{\dagger} \hat{a}$.

Consequently, we can describe the dynamics of the system through equations of the form

$$
\begin{aligned}
\dot{\hat{a}}= & -\left(\kappa+i \delta_{\mathrm{cA}}\right) \hat{a} \\
& -i e^{-i \phi_{\mathrm{A}}} \sum_{j} \Omega_{j} \hat{b}_{x j}-\sqrt{2 \kappa} e^{-i \nu_{x} t} \hat{a}_{\mathrm{in}}(t), \\
\dot{\hat{b}}_{x j}= & -i \nu_{x} \hat{b}_{x j}-i e^{i \phi_{\mathrm{A}}} \Omega_{j} \hat{a},
\end{aligned}
$$

with

$$
\Omega_{j}=-\frac{\eta_{x} g_{0} \mathcal{E}_{\mathrm{A}}}{\Delta_{0 \mathrm{~A}}} \cos \left(\theta_{j}\right) .
$$

Adiabatic elimination of the cavity mode as before then leads to

$$
\begin{aligned}
\dot{\hat{b}}_{x j}= & -\left(\Gamma_{j}+i \nu_{x}\right) \hat{b}_{x j}-\sum_{k \neq j} \frac{\Omega_{j} \Omega_{k}}{\kappa} \hat{b}_{x k} \\
& +e^{i \phi_{\mathrm{A}}} \sqrt{2 \Gamma_{j}} e^{-i \nu_{x} t} \hat{a}_{\mathrm{in}}(t),
\end{aligned}
$$

where $\Gamma_{j}=\Omega_{j}^{2} / \kappa$. So, through the laser and cavity fields one realizes a coupling between the motional modes of different atoms.

Now, defining the "collective" mode operator

$$
\hat{B}_{x}=\frac{1}{\sqrt{N_{\mathrm{eff}}}} \sum_{k} \cos \left(\theta_{k}\right) \hat{b}_{x k}
$$

with $N_{\text {eff }}=\sum_{j} \cos ^{2}\left(\theta_{j}\right)$ (such that $\left.\left[\hat{B}_{x}, \hat{B}_{x}^{\dagger}\right]=1\right)$, one finds

$$
\dot{\hat{B}}_{x}=-\left(N_{\mathrm{eff}} \Gamma+i \nu_{x}\right) \hat{B}_{x}-\sqrt{2 N_{\mathrm{eff}} \Gamma} e^{-i \nu_{x} t} \hat{a}_{\mathrm{in}}(t),
$$

with $\Gamma=\left(\eta_{x} g_{0} \mathcal{E}_{\mathrm{A}} / \Delta_{0 \mathrm{~A}}\right)^{2} / \kappa$. This points to the possibility of using the collective mode for quantum state transfer and storage [48. A potential advantage of such an approach is the enhanced effective transfer rate $N_{\text {eff }} \Gamma$, which (for $N_{\text {eff }}>1$ ) should enable the requirement of strong-coupling cavity QED to be relaxed somewhat.

The possibility also arises for the preparation of some very interesting many-atom entangled states. For example, motional state transfer of a Schrödinger Cat state $\mathcal{N}_{+}^{-1}\left(|\alpha\rangle_{x}+|-\alpha\rangle_{x}\right)$ to the collective mode, perhaps from a single-atom (cavity QED) source, would result in a manyatom state of the form (omitting the normalization)

$$
\left|\alpha_{1}, \alpha_{2}, \alpha_{3}, \ldots\right\rangle_{x}+\left|-\alpha_{1},-\alpha_{2},-\alpha_{3}, \ldots\right\rangle_{x},
$$

where $\alpha_{j}=\alpha \cos \left(\theta_{j}\right) / \sqrt{N_{\text {eff }}}$.

\section{E. Coupling to collective trapped ion modes}

Earlier, we discussed a scheme for coupling orthogonal single-ion and many-ion collective vibrational modes using a pair of laser fields. This scheme could also be utilized in the context of state transfer and storage, albeit somewhat indirectly, as depicted in Fig. 7. In particular, state transfer between motion and light and from one cavity location to another could be achieved using a single-ion interaction with the field mode (assuming that individual addressing of the ion by the coupling laser is possible), after which the ion-cavity coupling is switched off and the coupling between the single-ion mode and a particular collective motional mode is switched on (for example, to transfer a quantum state from the single-ion mode to the collective mode). Storage of quantum information in certain collective vibrational modes could be advantageous due to the very slow heating rates experienced by these modes [49]. 


\section{ENTANGLING DISTANT ATOMS}

Having described and analysed basic techniques for preparing "local" entanglement of vibrational modes, and then for coupling vibrational modes to propagating light fields, we now want to outline some specific and interesting possibilities for distribution of entanglement between distant locations. The procedure is straightforward: one prepares an entangled state of the orthogonal vibrational modes in the $x$ - and, say, $z$-directions, after which the $x$ mode is coupled to the cavity and thence to the external light field. With appropriate time-dependent coupling we have seen that the properties of the $x$ mode can be transferred with high fidelity to the corresponding mode of a distant cavity-confined atom (or atoms). That is, we can perform the transformation

$$
\begin{aligned}
& \left(\sum_{n, m} c_{n m}|n\rangle_{x}^{(1)} \otimes|m\rangle_{z}^{(1)}\right) \otimes|0\rangle_{x}^{(2)} \\
& \rightarrow|0\rangle_{x}^{(1)} \otimes\left(\sum_{n, m} c_{n m}|n\rangle_{x}^{(2)} \otimes|m\rangle_{z}^{(1)}\right),
\end{aligned}
$$

where $|n\rangle_{x, z}^{(i)}$ is a vibrational Fock state of the atom at location $i$. Hence, the $x$ and $z$ modes of the atoms at locations 2 and 1 , respectively, become entangled.

Note that theoretical proposals exist for schemes that would in principle allow the preparation of arbitrary two-dimensional vibrational states of a trapped atom 22 24,26. It follows that, using the state transfer scheme, it would in principle be possible to prepare an arbitrary entangled state of distantly-separated atoms. Below we highlight briefly just a few examples of particular interest, with emphasis on possibilities associated with the particular two-mode entangling schemes presented in Section II.

\section{A. Examples}

\section{EPR state preparation between distant atoms}

Here, we use the scheme of Section II.A.2 to prepare the state $\left|\psi_{\mathrm{sq}}(r)\right\rangle^{(1)}$ given in (14), following which the state transfer operation outlined above produces the delocalized state

$$
\cosh ^{-1}(r) \sum_{m=0}^{\infty}[-\tanh (r)]^{m}|m\rangle_{x}^{(2)} \otimes|m\rangle_{z}^{(1)} .
$$

The corresponding Wigner function is

$$
\begin{aligned}
W & \left(\tilde{x}^{(2)}, \tilde{p}_{x}^{(2)} ; \tilde{z}^{(1)}, \tilde{p}_{z}^{(1)}\right) \\
= & \frac{4}{\pi^{2}} \exp \left\{-\left[\left(\tilde{x}^{(2)}+\tilde{z}^{(1)}\right)^{2}+\left(\tilde{p}_{x}^{(2)}-\tilde{p}_{z}^{(1)}\right)^{2}\right] e^{+2 r}\right\} \\
& \times \exp \left\{-\left[\left(\tilde{x}^{(2)}-\tilde{z}^{(1)}\right)^{2}+\left(\tilde{p}_{x}^{(2)}+\tilde{p}_{z}^{(1)}\right)^{2}\right] e^{-2 r}\right\},
\end{aligned}
$$

where $\left\{\tilde{z}^{(1)}, \tilde{p}_{z}^{(1)}\right\}$ and $\left\{\tilde{x}^{(2)}, \tilde{p}_{x}^{(2)}\right\}$ are position and momentum variables for the atoms at locations 1 and 2 , respectively.

So, one prepares an EPR state in position and momentum of a pair of (distantly) separated atoms. Apart from being of great historical significance, this state also constitutes the essential resource for the teleportation of continuous variables [11,50,51]. In particular, in the present context it offers the possibility of teleporting atomic center-of-mass wave functions between distant sites [14].

\section{Delocalized mesoscopic states}

As mentioned earlier, the linear mixing operation described by (8) can also generate entanglement given suitable initial states. Consider, for example, the situation in which, say, the $z$ mode is initially prepared in its ground state while the $x$ mode is prepared in a Schrödinger Cat state, i.e.,

$$
|\psi(0)\rangle=\frac{1}{\mathcal{N}_{+}}\left(|\alpha\rangle_{x}^{(1)}+|-\alpha\rangle_{x}^{(1)}\right) \otimes|0\rangle_{z}^{(1)} .
$$

Application of the linear mixing operation for a time $T=$ $\pi /(4 \chi)$ will transform this into the state

$$
\begin{aligned}
|\psi(T)\rangle=\frac{1}{\mathcal{N}_{+}} & \left(\left|\frac{\alpha}{\sqrt{2}}\right\rangle_{x}^{(1)} \otimes\left|\frac{-\alpha}{\sqrt{2}}\right\rangle_{z}^{(1)}\right. \\
& \left.+\left|\frac{-\alpha}{\sqrt{2}}\right\rangle_{x}^{(1)} \otimes\left|\frac{\alpha}{\sqrt{2}}\right\rangle_{z}^{(1)}\right) .
\end{aligned}
$$

The state transfer procedure then generates the delocalized state

$$
\frac{1}{\mathcal{N}_{+}}\left(\left|\frac{\alpha}{\sqrt{2}}\right\rangle_{x}^{(2)} \otimes\left|\frac{-\alpha}{\sqrt{2}}\right\rangle_{z}^{(1)}+\left|\frac{-\alpha}{\sqrt{2}}\right\rangle_{x}^{(2)} \otimes\left|\frac{\alpha}{\sqrt{2}}\right\rangle_{z}^{(1)}\right)
$$

From what we have seen in our numerical analysis, the coherent state amplitude $\alpha$ can be reasonably large (while still allowing high fidelity operations and transfers), and so the entangled state (81) could be regarded as a delocalized mesoscopic state [52].

Schemes have also been proposed for preparing a state of the form

$$
\frac{1}{\sqrt{2}}\left(|N\rangle_{x}^{(1)} \otimes|0\rangle_{z}^{(1)}+|0\rangle_{x}^{(1)} \otimes|N\rangle_{z}^{(1)}\right)
$$

where $|N\rangle_{x}^{(1)}$ is a Fock state (see, for example, 25), which would lead to a delocalized state

$$
\frac{1}{\sqrt{2}}\left(|N\rangle_{x}^{(2)} \otimes|0\rangle_{z}^{(1)}+|0\rangle_{x}^{(2)} \otimes|N\rangle_{z}^{(1)}\right)
$$


Such states are of potential interest in the context of phase sensitivity in a two-mode interferometer, where they should allow measurements at the Heisenberg uncertainty limit (see, for example, [25], and references therein).

\section{Many-atom entangled states}

Through the many-atom configurations discussed in Sections III.D and III.E one can generalize the entanglement distribution procedure to collections of trapped atoms or ions located at the distant sites. For example, one could imagine entangling collective vibrational modes of two strings of trapped ions at separate locations. Such schemes complement proposals for entangling collective internal atomic states of separated free-space atomic ensembles using propagating light fields (see, for example, 53 56]).

\section{CONCLUSION}

In this work we have described and analysed schemes (i) for producing motional state entanglement of an atom (or atoms) at one location and (ii) for distributing this entanglement between atoms at distant locations. The particular example we have focussed on for generating (local) entanglement was chosen for its relative simplicity and for its close relation to schemes already implemented in the laboratory. A further motivating factor which should also be emphasized is that the operations that it allows are of direct relevance to quantum communication and computing with continuous variables, for which squeezers and linear mixers are basic elements (see, for example, [11,51,57,60]).

The cavity-QED-based motional state transfer scheme that enables the entanglement to be distributed between distant sites has been introduced elsewhere [12], but the present work extends significantly the numerical analysis of this scheme. In particular, we have considered the transfer of what may be regarded as mesoscopic motional states and from this we have been able to gauge the validity of some of the more fundamental assumptions implicit in the scheme, such as the Lamb-Dicke and rotating-wave approximations. Our calculations suggest that states of a substantial "size" can be transferred with high fidelity for physically reasonable parameter values and we have discussed some possible experimental scenarios, encouraged by spectacular recent advances in atom and ion trapping technology and in cavity QED.

The possibilities offered by the schemes for distributed entanglement are many and varied, and we have discussed just a few examples in Section IV, along with some possible applications such as quantum teleportation of atomic wavepackets. Of course, in addition to potential applications in quantum communication and computing, entangled and delocalized states of the form considered here would offer some unique and fascinating opportunities for fundamental tests of quantum mechanics versus local realism using massive particles [13,52,61].

\section{ACKNOWLEDGMENTS}

ASP thanks H.J. Kimble, H. Ritsch, I. Cirac, and D. Leibfried for discussions and comments and gratefully acknowledges support from the Marsden Fund of the Royal Society of New Zealand. ASP also thanks the Quantum Optics groups at the University of Innsbruck and the California Institute of Technology for support and hospitality during visits when part of this work was carried out.

\section{APPENDIX A: EFFECTS OF ATOMIC SPONTANEOUS EMISSION}

\section{Motional state preparation}

Clearly, a very important assumption is that the effects of atomic spontaneous emission can be neglected. In a master equation approach, atomic spontaneous emission with the effects of recoil taken into account is modelled by a term of the form (considering, for simplicity, motion only along the $x$ axis) [62]

$$
\{\dot{\hat{\rho}}\}_{\text {spon }}=\frac{\gamma}{2}\left(2 \hat{\sigma}_{-} \tilde{\rho} \hat{\sigma}_{+}-\hat{\sigma}_{+} \hat{\sigma}_{-} \hat{\rho}-\hat{\rho} \hat{\sigma}_{+} \hat{\sigma}_{-}\right)
$$

where

$$
\begin{aligned}
\tilde{\rho} & =\frac{1}{2} \int_{-1}^{+1} d u W(u) e^{i k u \hat{x}} \hat{\rho} e^{-i k u \hat{x}} \\
& =\frac{1}{2} \int_{-1}^{+1} d u W(u) e^{i u \eta_{x}\left(\hat{b}_{x}+\hat{b}_{x}^{\dagger}\right)} \hat{\rho} e^{-i u \eta_{x}\left(\hat{b}_{x}+\hat{b}_{x}^{\dagger}\right)} .
\end{aligned}
$$

Here, $\gamma$ is the spontaneous emission rate and $W(u)=$ $(3 / 4)\left(1+u^{2}\right)$ describes the angular distribution of spontaneous emission for an atomic dipole transition.

Staying in one dimension and considering the preparation of a squeezed state of the motion, the appropriate combination of laser fields is

$$
\begin{aligned}
E_{\mathrm{L}}(\hat{x}, t) & =E_{1}(\hat{x}, t)+E_{2}(\hat{x}, t) \\
& =\frac{\mathcal{E}}{\sqrt{2}}\left[e^{-i \eta_{x}\left(\hat{b}_{x}+\hat{b}_{x}^{\dagger}\right)}+e^{-i \delta_{21} t+i \eta_{x}\left(\hat{b}_{x}+\hat{b}_{x}^{\dagger}\right)}\right],
\end{aligned}
$$

with $\delta_{21}=2 \nu_{x}$. Adiabatically eliminating the atomic excited state, which amounts to setting

$$
\hat{\sigma}_{-} \simeq i \frac{E_{\mathrm{L}}(\hat{x}, t)}{\Delta_{01}}
$$

in (A1), one finds that the leading order (in $\eta_{x}$ ) contribution to the motional dynamics arising from atomic recoil due to spontaneous emission takes the form 


$$
\begin{gathered}
s(t)\left[2\left(\hat{b}_{x}+\hat{b}_{x}^{\dagger}\right) \hat{\rho}\left(\hat{b}_{x}+\hat{b}_{x}^{\dagger}\right)-\left(\hat{b}_{x}+\hat{b}_{x}^{\dagger}\right)^{2} \hat{\rho}\right. \\
\left.-\hat{\rho}\left(\hat{b}_{x}+\hat{b}_{x}^{\dagger}\right)^{2}\right]
\end{gathered}
$$

where

$$
s(t)=\frac{1}{4} \frac{\gamma \eta_{x}^{2} \mathcal{E}^{2}}{\Delta_{01}^{2}}\left(\left|1-e^{-i \delta_{21} t}\right|^{2}+\frac{1}{5}\left|1+e^{i \delta_{21} t}\right|^{2}\right) .
$$

So, the rate at which atomic spontaneous emission can be expected to influence the motional dynamics is on the order of $\gamma \eta_{x}^{2} \mathcal{E}^{2} / \Delta_{01}^{2}$, whereas the rate at which the squeezed state is prepared is essentially $\chi=4 \eta_{x}^{2} \mathcal{E}^{2} / \Delta_{01}$, leading to the condition

$$
\frac{\gamma}{\Delta_{01}} \ll 1
$$

for spontaneous emission to have negligible effect.

\section{Motion-light coupling}

The analysis of the effects of spontaneous emission in the context of the atom-cavity state transfer scheme follows the above working, only now the relevant substitution is

$$
\hat{\sigma}_{-} \simeq-\frac{\mathcal{E}_{\mathrm{A}}(t)}{\Delta_{0 \mathrm{~A}}}-\frac{g_{0}}{\Delta_{0 \mathrm{~A}}} \sin (k \hat{x}) \hat{a}
$$

Assuming that $\mathcal{E}_{\mathrm{A}} \gg \eta_{x} g_{0} \sqrt{\left\langle\hat{a}^{\dagger} \hat{a}\right\rangle}$, to leading order in $\eta_{x}$ one obtains a term of the form

$$
\begin{aligned}
\eta_{x}^{2} \frac{\gamma}{10} \frac{\mathcal{E}_{\mathrm{A}}^{2}}{\Delta_{0 \mathrm{~A}}^{2}}\left[2\left(\hat{b}_{x}+\hat{b}_{x}^{\dagger}\right) \hat{\rho}\left(\hat{b}_{x}+\hat{b}_{x}^{\dagger}\right)-\left(\hat{b}_{x}+\hat{b}_{x}^{\dagger}\right)^{2} \hat{\rho}\right. \\
\left.-\hat{\rho}\left(\hat{b}_{x}+\hat{b}_{x}^{\dagger}\right)^{2}\right] .
\end{aligned}
$$

Hence, in order to be able to neglect the effects of spontaneous emission on the transfer process, one requires that

$$
\Gamma=\frac{\eta_{x}^{2} g_{0}^{2} \mathcal{E}_{\mathrm{A}}^{2}}{\kappa \Delta_{0 \mathrm{~A}}^{2}} \gg \eta_{x}^{2} \frac{\gamma}{10} \frac{\mathcal{E}_{\mathrm{A}}^{2}}{\Delta_{0 \mathrm{~A}}^{2}} \quad \text { or } \quad \frac{10 g_{0}^{2}}{\kappa \gamma} \gg 1 .
$$

This, not surprisingly, corresponds to the regime of strong coupling in cavity QED.

\section{APPENDIX B: LAMB-DICKE APPROXIMATION FOR THE ATOM-CAVITY COUPLING LASER}

In deriving our model for motion-light coupling we make the (simplifying) assumption that position dependence of the external coupling laser can be neglected, i.e., $E_{\mathrm{A}}(\hat{y}, t) \simeq \mathcal{E}_{\mathrm{A}}(t) e^{-i \phi_{\mathrm{A}}}$. We mentioned that this could be justified, for instance, in the case where the laser field forms a standing wave with the trap centered at an antinode, i.e., $E_{\mathrm{A}}(\hat{y}, t) \propto \cos (k \hat{y}) \simeq 1$, assuming tight confinement in the $y$-direction.

However, a traveling wave laser field (which is most likely a simpler proposition from an experimental point of view) should also suffice, as the following argument shows. Assume that

$$
E_{\mathrm{A}}(\hat{y}, t)=\mathcal{E}_{\mathrm{A}}(t) e^{i k \hat{y}}
$$

Then, first of all, $\left|E_{\mathrm{A}}(\hat{y}, t)\right|^{2}=\mathcal{E}_{\mathrm{A}}(t)^{2}$. Secondly, on adiabatically eliminating the atomic excited state, we have an interaction term of the form (omitting constant coefficients)

$$
e^{i \eta_{y}\left(\hat{b}_{y}+\hat{b}_{y}^{\dagger}\right)} \sin \left[\eta_{x}\left(\hat{b}_{x}+\hat{b}_{x}^{\dagger}\right)\right]\left(\hat{a}+\hat{a}^{\dagger}\right) .
$$

To second order in the Lamb-Dicke parameters, this expression takes the form

$$
\begin{aligned}
& \eta_{x}\left(\hat{b}_{x}+\hat{b}_{x}^{\dagger}\right)\left(\hat{a}+\hat{a}^{\dagger}\right) \\
& \quad+i \eta_{x} \eta_{y}\left(\hat{b}_{y}+\hat{b}_{y}^{\dagger}\right)\left(\hat{b}_{x}+\hat{b}_{x}^{\dagger}\right)\left(\hat{a}+\hat{a}^{\dagger}\right) .
\end{aligned}
$$

Now, we have $\delta_{\mathrm{cA}}=\nu_{x}$, and assuming that $\nu_{x}$ and $\nu_{y}$ are both large, and that $\left|2 \nu_{x}-\nu_{y}\right|$ is also large, the only contribution in (B3) that is not rapidly rotating is the desired term $\eta_{x}\left(\hat{a}^{\dagger} \hat{b}_{x}+\hat{b}_{x}^{\dagger} \hat{a}\right)$. That is, the contribution from the position dependence of $E_{\mathrm{A}}(\hat{y}, t)$, as well as being of order $\eta_{x} \eta_{y}$, is also rapidly rotating (assuming large $\nu_{y}$ ), and hence can be neglected.

[1] M. Brune et al., Phys. Rev. Lett. 72, 3339 (1994); G. Nogues et al., Nature 400, 239 (1999).

[2] Q.A. Turchette et al., Phys. Rev. Lett. 75, 4710 (1995).

[3] C. Monroe et al., Phys. Rev. Lett. 75, 4714 (1995).

[4] A. Rauschenbeutel et al., Phys. Rev. Lett. 83, 5166 (1999).

[5] E. Hagley et al., Phys. Rev. Lett. 79, 1 (1997).

[6] Q.A. Turchette et al., Phys. Rev. Lett. 81, 3631 (1998).

[7] A. Rauschenbeutel et al., Science 288, 2024 (2000).

[8] C.A. Sackett et al., Nature 404, 256 (2000).

[9] D. Bouwmeester et al., Nature 390, 575 (1997).

[10] D. Boschi et al., Phys. Rev. Lett. 80, 1121 (1998).

[11] A. Furusawa et al.., Science 282, 706 (1998).

[12] A.S. Parkins and H.J. Kimble, J. Opt. B: Quantum Semiclass. Opt. 1, 496 (1999).

[13] A.S. Parkins and H.J. Kimble, Phys. Rev. A 61, 052104 (2000).

[14] A.S. Parkins and H.J. Kimble, in Frontiers of Laser Physics and Quantum Optics, Proceedings of the International Conference on Laser Physics and Quantum Optics, eds. Z. Xu, S. Xie, S.-Y. Zhu, and M.O. Scully (Springer, Berlin, 2000), p.321. See also quant-ph/9909021.

[15] D.M. Meekhof et al., Phys. Rev. Lett. 76, 1796 (1996). 
[16] C. Monroe et al., Science 272, 1131 (1996).

[17] D.J. Wineland et al., Jou. Res. Nat. Inst. Stand. Tech. 103, 259 (1998).

[18] Ch. Roos et al., Phys. Rev. Lett. 83, 4713 (1999); F. Schmidt-Kaler et al., quant-ph/0003096, submitted to J. Mod. Opt..

[19] S.-C. Gou and P.L. Knight, Phys. Rev. A 54, 1682 (1996).

[20] S.-C. Gou, J. Steinbach, and P.L. Knight, Phys. Rev. A 54, R1014 (1996); ibid. 54, 4315 (1996).

[21] J. Steinbach, J. Twamley, and P.L. Knight, Phys. Rev. A 56, 4815 (1997).

[22] S.A. Gardiner, J.I. Cirac, and P. Zoller, Phys. Rev. A 55, 1683 (1997).

[23] G. Drobný and B. Hladký, Acta Phys. Slov. 47, 277 (1997).

[24] G. Drobný, B. Hladký, and V. Bužek, Phys. Rev. A 58, 2481 (1998).

[25] D.J. Wineland et al., Physica Scripta T76, 147 (1998).

[26] B. Kneer and C.K. Law, Phys. Rev. A 57, 2096 (1998).

[27] D.F. Walls and G.J. Milburn, Quantum Optics (SpringerVerlag, Berlin, 1994).

[28] Z.Y. Ou, S.F. Pereira, and H.J. Kimble, Appl. Phys. B 55, 265 (1992).

[29] A. Einstein, B. Podolsky, and N. Rosen, Phys. Rev. 47, 777 (1935).

[30] See, for example, M.G. Raizen et al., Phys. Rev. A 45, 6493 (1992); I. Waki et al., Phys. Rev. Lett. 68, 2007 (1992); H.C. Nägerl et al., Appl. Phys. B: Photophys. Laser Chem. 66, 603 (1998); R.J. Hughes et al., Fortschr. Phys. 46, 329 (1998).

[31] J.I. Cirac and P. Zoller, Phys. Rev. Lett. 74, 4091 (1995).

[32] D.F.V. James, Appl. Phys. B 66, 181 (1998).

[33] G. Morigi, J. Eschner, J.I. Cirac, and P. Zoller, Phys. Rev. A 59, 3797 (1999).

[34] H. Zeng and F. Lin, Phys. Rev. A 50, R3589 (1994).

[35] Note that the terms of second order in $\eta_{x}$ correspond to a nonlinear coupling between the modes which can, in a slightly different configuration [in particular, with the coupling laser turned off, i.e., $\Omega(t)=0$, and with the cavity mode driven by a coherent field] be used for a quantum nondemolition measurement of the phonon number; see F.E. Harrison, A.S. Parkins, M.J. Collett, and D.F. Walls, Phys. Rev. A 55, 4412 (1997).

[36] C.W. Gardiner and P. Zoller, Quantum Noise (SpringerVerlag, Berlin, 2000).

[37] J.I. Cirac, P. Zoller, H.J. Kimble, and H. Mabuchi, Phys. Rev. Lett. 78, 3221 (1997).

[38] C.W. Gardiner, Phys. Rev. Lett. 70, 2269 (1993).

[39] H.J. Carmichael, Phys. Rev. Lett. 70, 2273 (1993).

[40] P.T. Cochrane, G.J. Milburn, and W.J. Munro, Phys. Rev. A 59, 2631 (1999); M.C. Oliveira and W.J. Munro, Phys. Rev. A 61, 042309 (2000).

[41] Y. Nambu et al., Phys. Rev. A 62, 012312 (2000).

[42] Q.A. Turchette et al., Phys. Rev. A 61, 063418 (2000).

[43] J. Ye, D.W. Vernooy, and H.J. Kimble, Phys. Rev. Lett. 83, 4987 (1999).

[44] J. Fortagh et al., Appl. Phys. B 70, 701 (2000); D. Cassettari et al., Appl. Phys. B 70, 721 (2000).

[45] C.J. Hood et al., Science 287, 1447 (2000).

[46] P.W.H. Pinkse et al., Nature 404, 365 (2000).
[47] G. Rempe et al., Opt. Lett. 17, 363 (1992).

[48] Note that with a vacuum input to the cavity field, the dynamics described by Eq.(74) will correspond simply to cooling of the collective mode to its ground state. In this way, the collective mode could be "initialized".

[49] B.E. King et al., Phys. Rev. Lett. 81, 1525 (1998). In this experiment with a pair of ${ }^{9} \mathrm{Be}^{+}$ions, the heating rates of the modes of relative ion motion (for example, the "stretch" mode) were found to be significantly smaller than those of the center-of-mass modes

[50] L. Vaidman, Phys. Rev. A 49, 1473 (1994). See also L. Vaidman and N. Yoran, Phys. Rev. A 59, 116 (1999).

[51] S.L. Braunstein and H.J. Kimble, Phys. Rev. Lett. 80, 869 (1998).

[52] A.S. Parkins, quant-ph/0006113, submitted to J. Opt. B: Quantum Semiclass. Opt.. In the proposal presented in this work, motion in only one dimension is considered, but auxiliary internal atomic states that are not coupled to the cavity field, and thereby do not participate in the motional state transfer process, enable delocalized coherent or Fock states to be prepared.

[53] E.S. Polzik, Phys. Rev. A 59, 4202 (1999).

[54] M.D. Lukin, S.F. Yelin, and M. Fleischhauer, Phys. Rev. Lett. 84, 4232 (2000).

[55] A.E. Kozhekin, K. Mølmer, and E.S. Polzik, Phys. Rev. A 62, 033809 (2000).

[56] L.M. Duan et al., quant-ph/0003111.

[57] S. Lloyd and S.L. Braunstein, Phys. Rev. Lett. 82, 1784 (1999).

[58] P. van Loock and S.L. Braunstein, Phys. Rev. Lett. 84, 3482 (2000).

[59] S.L. Braunstein and H.J. Kimble, Phys. Rev. A 61, 042302 (2000).

[60] T.C. Ralph, Phys. Rev. A 61, 010303(R) (2000).

[61] E.S. Fry and T. Walther, Adv. At. Mol. Phys. 42, 1 (2000).

[62] See, for example, J. I. Cirac et al., Phys. Rev. A 46, 2668 (1992); C. D'Helon and G. J. Milburn, ibid. 52, 4755 (1995).

TABLE I. Two-mode squeezed state preparation: examples of fidelities achievable for several different trapping configurations. Note that, in the dimensionless units used here, the values of $\chi$ correspond to the choice $\mathcal{E}^{2} / \Delta_{01}=0.1$. Also, since $\eta_{i} \propto 1 / \sqrt{\nu_{i}}$, setting $\eta_{x}^{\prime}=\eta_{z}^{\prime}$ means that $(\alpha / \beta)=\sqrt{\nu_{x} / \nu_{z}}$.

\begin{tabular}{cccccc}
\hline \hline$\eta_{x}^{\prime}, \eta_{z}^{\prime}$ & $\nu_{x}$ & $\nu_{z}$ & $\chi$ & $r=\chi T$ & $F$ \\
\hline 0.1 & 1 & 3 & 0.004 & 1 & 0.991 \\
0.1 & 1 & 3 & 0.004 & 1.5 & 0.932 \\
0.1 & 1 & 4 & 0.004 & 1.5 & 0.955 \\
0.0707 & 1 & 3 & 0.002 & 1 & 0.996 \\
0.0707 & 1 & 3 & 0.002 & 1.5 & 0.975 \\
0.0707 & 1 & 4 & 0.002 & 1.5 & 0.986 \\
0.0577 & 1 & 3 & 0.00133 & 1 & 0.998 \\
0.0577 & 1 & 3 & 0.00133 & 1.5 & 0.987 \\
0.0577 & 1 & 4 & 0.00133 & 1.5 & 0.994 \\
\hline \hline
\end{tabular}


TABLE II. Numerical results for transfer of the truncated phase state $1 / \sqrt{11} \sum_{n=0}^{10}|n\rangle_{x}$. Parameters are $\kappa=1$, $g_{0}^{2} / \Delta_{0 \mathrm{~A}}=0.2,\left(g_{0} \mathcal{E}_{\mathrm{A}}^{\max }\right) / \Delta_{0 \mathrm{~A}}=1.0$.

\begin{tabular}{ccc}
\hline \hline$\eta_{x}$ & $\nu_{x}\left(=\delta_{\mathrm{cA}}\right)$ & $\left\langle\Psi\left(t_{\mathrm{f}}\right) \mid \Psi\left(t_{\mathrm{f}}\right)\right\rangle$ (no jump) \\
\hline 0.1 & 5 & 0.65 \\
0.1 & 10 & 0.90 \\
0.1 & 20 & 0.96 \\
0.0707 & 5 & 0.66 \\
0.0707 & 10 & 0.91 \\
0.0707 & 20 & 0.97 \\
\hline \hline
\end{tabular}

TABLE III. Numerical results for transfer of the truncated phase state $1 / \sqrt{21} \sum_{n=0}^{20}|n\rangle_{x}$. Parameters are $\kappa=1$, $g_{0}^{2} / \Delta_{0 \mathrm{~A}}=0.2,\left(g_{0} \mathcal{E}_{\mathrm{A}}^{\max }\right) / \Delta_{0 \mathrm{~A}}=1.0$.

\begin{tabular}{ccc}
\hline \hline$\eta_{x}$ & $\nu_{x}\left(=\delta_{\mathrm{cA}}\right)$ & $\left\langle\Psi\left(t_{\mathrm{f}}\right) \mid \Psi\left(t_{\mathrm{f}}\right)\right\rangle($ no jump) \\
\hline 0.1 & 10 & 0.79 \\
0.1 & 20 & 0.88 \\
0.0707 & 10 & 0.84 \\
0.0707 & 20 & 0.94 \\
\hline \hline
\end{tabular}

TABLE IV. Numerical results for transfer of the Fock state $|n=10\rangle_{x}$. Parameters are $\kappa=1, g_{0}^{2} / \Delta_{0 \mathrm{~A}}=0.2$, $\left(g_{0} \mathcal{E}_{\mathrm{A}}^{\max }\right) / \Delta_{0 \mathrm{~A}}=1.0$.

\begin{tabular}{ccc}
\hline \hline$\eta_{x}$ & $\nu_{x}\left(=\delta_{\mathrm{cA}}\right)$ & $\left\langle\Psi\left(t_{\mathrm{f}}\right) \mid \Psi\left(t_{\mathrm{f}}\right)\right\rangle$ \\
\hline 0.1 & 10 & 0.82 \\
0.1 & 20 & 0.92 \\
0.0707 & 10 & 0.85 \\
0.0707 & 20 & 0.95 \\
\hline \hline
\end{tabular}

TABLE V. Numerical results for transfer of the Schrödinger Cat state $\mathcal{N}_{+}^{-1}\left(|\alpha\rangle_{x}+|-\alpha\rangle_{x}\right)$, with $\alpha=\sqrt{10}$. Parameters are $\kappa=1, g_{0}^{2} / \Delta_{0 \mathrm{~A}}=0.2,\left(g_{0} \mathcal{E}_{\mathrm{A}}^{\max }\right) / \Delta_{0 \mathrm{~A}}=1.0$.

\begin{tabular}{ccc}
\hline \hline$\eta_{x}$ & $\nu_{x}\left(=\delta_{\mathrm{cA}}\right)$ & $\left\langle\Psi\left(t_{\mathrm{f}}\right) \mid \Psi\left(t_{\mathrm{f}}\right)\right\rangle$ (no jump) \\
\hline 0.1 & 10 & 0.81 \\
0.1 & 20 & 0.91 \\
0.0707 & 10 & 0.85 \\
0.0707 & 20 & 0.95 \\
\hline \hline
\end{tabular}

FIG. 1. (a) Coordinate axes and laser configuration for two-dimensional motional state manipulation of a trapped atom. (The trapping potential is not shown.) (b) Excitation scheme for the linear mixer interaction. (c) Excitation scheme for preparation of a two-mode squeezed state of the motion. Only the first few vibrational levels are shown, and the excited atomic state is omitted for simplicity.

FIG. 2. Laser configuration for coupling two-ion collective and single-ion vibrational modes.

FIG. 3. Schematic of proposed (a) experimental setup and (b) excitation scheme for state transfer between the motion of a trapped atom or ion and a quantized cavity mode of the electromagnetic field. All input and output to the cavity mode is through just one mirror, i.e., the other mirror is assumed to be perfect.

FIG. 4. (a) Decay of the motional mode amplitude $\left|\left\langle\hat{b}_{x}(t)\right\rangle\right|$ with time, given an initial coherent state of the motion $|\alpha=\sqrt{10}\rangle_{x}$, with $\eta_{x}=0.1$ (lower solid line), 0.15 (upper solid line). The dashed curve is given by $\sqrt{10} \exp (-\Gamma t)$. Other parameters are $\kappa=1, \nu_{x}=\delta_{\mathrm{cA}}=10, g_{0}^{2} / \Delta_{0 \mathrm{~A}}=0.2$, and $\eta_{x} g_{0} \mathcal{E}_{\mathrm{A}} / \Delta_{0 \mathrm{~A}}=0.1$ (corresponding to $\Gamma=0.01$ ). Figures (b) and (c) show the cavity field amplitude $|\langle\hat{a}(t)\rangle|(\times 10)$ and the motional mode amplitude $\left|\left\langle\hat{b}_{x}(t)\right\rangle\right|$ for $\eta_{x}=0.1$ and $\eta_{x}=0.15$, respectively. Note that some of the structure (i.e., what looks like "beats") in $|\langle\hat{a}(t)\rangle|$ is due only to the finite number of points plotted.

FIG. 5. Fidelity $f(t)$ as a function of time for the parameters of Figure 4 with $\eta_{x}=0.1$ (circles), and $\eta_{x}=0.15$ (squares).

FIG. 6. Cascaded atom-cavity systems for motional state transfer. The coupling between cavities is assumed to be unidirectional (facilitated, for example, by Faraday isolators). During an ideal transfer no photons are detected by the photodetector, which monitors the output from the second cavity.

FIG. 7. Schematic for state transfer between light and collective motional modes of a string of trapped ions. (a) First, a single-ion interaction with the cavity field mode is used to "receive" an incoming quantum state, after which (b) auxiliary lasers couple the single-ion and collective vibrational modes. 
(a)

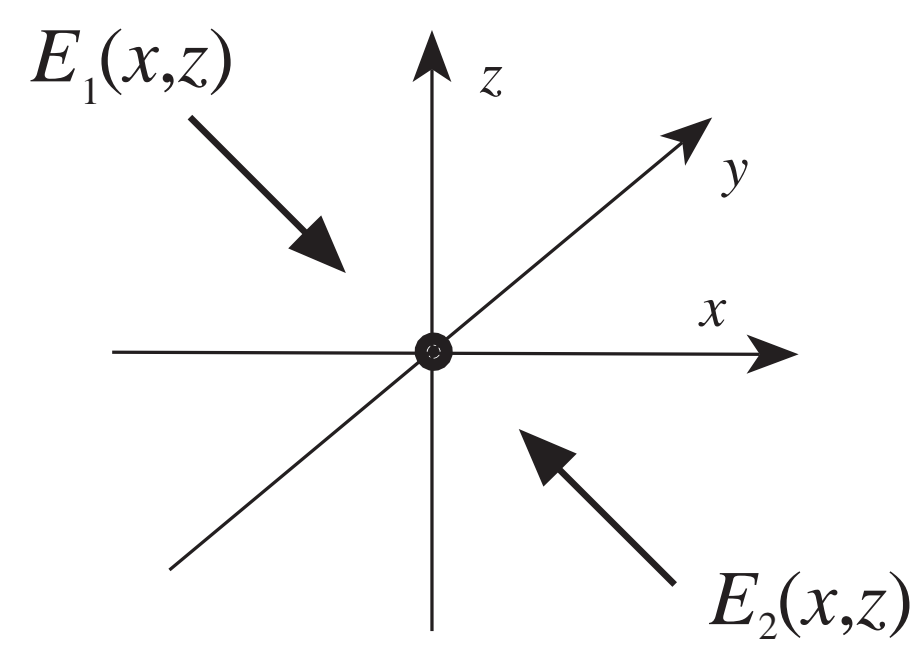

(b)

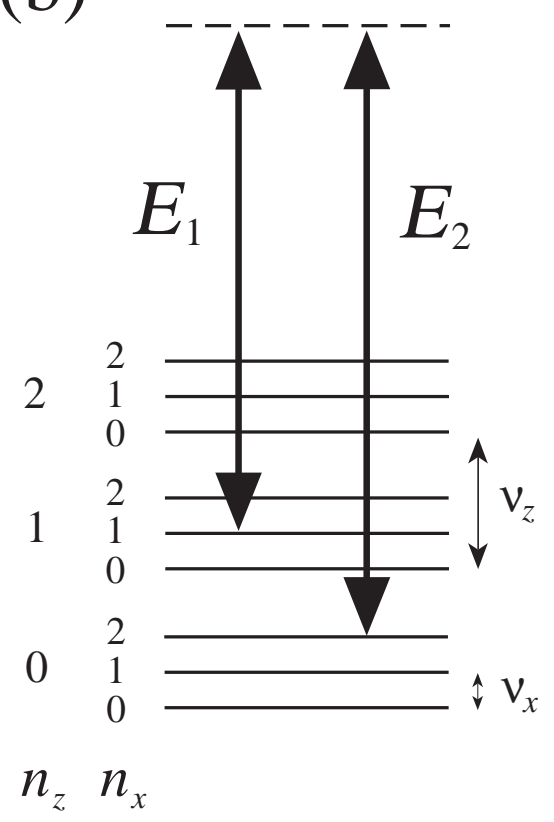

(c)

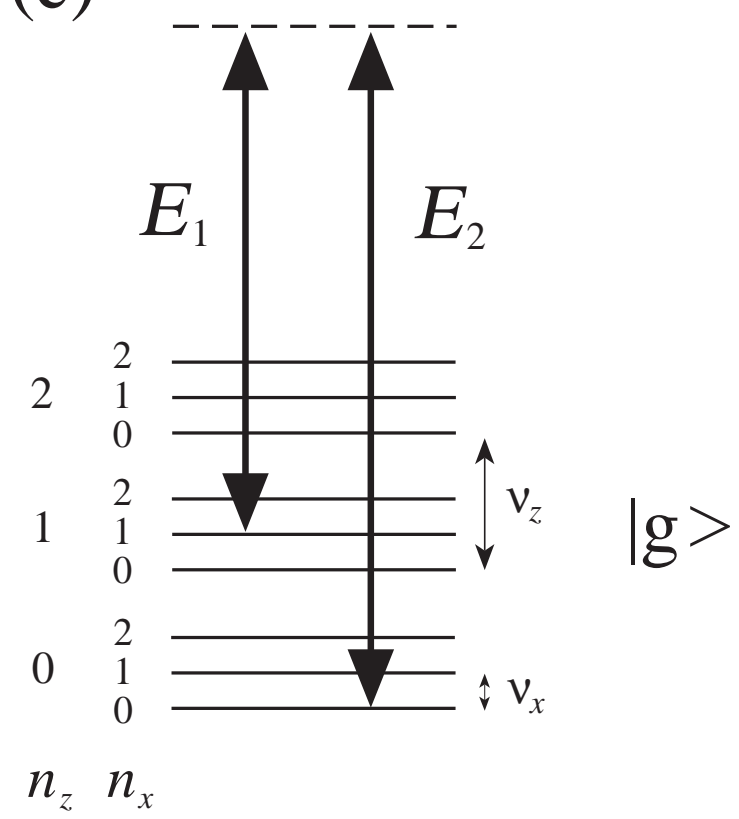

Figure 1 


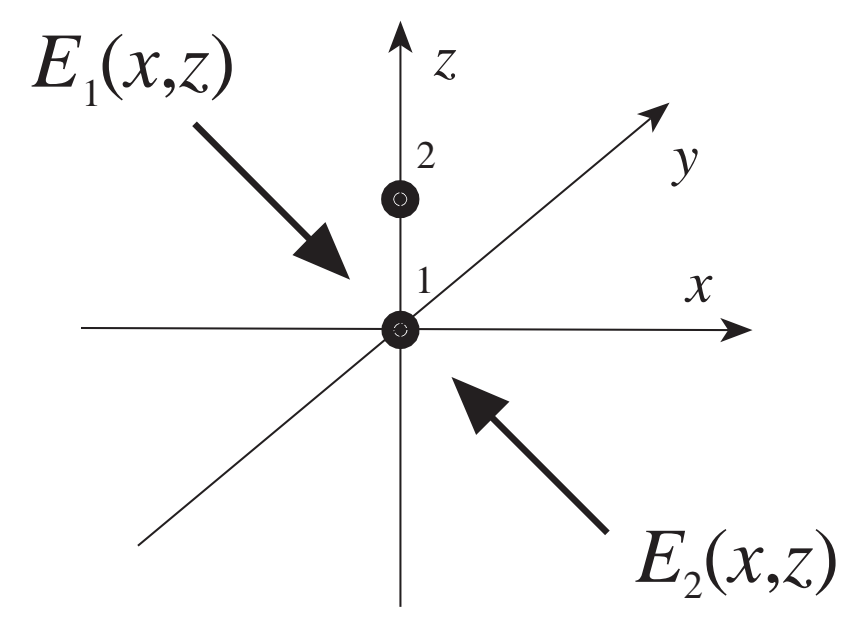

Figure 2 
(a)

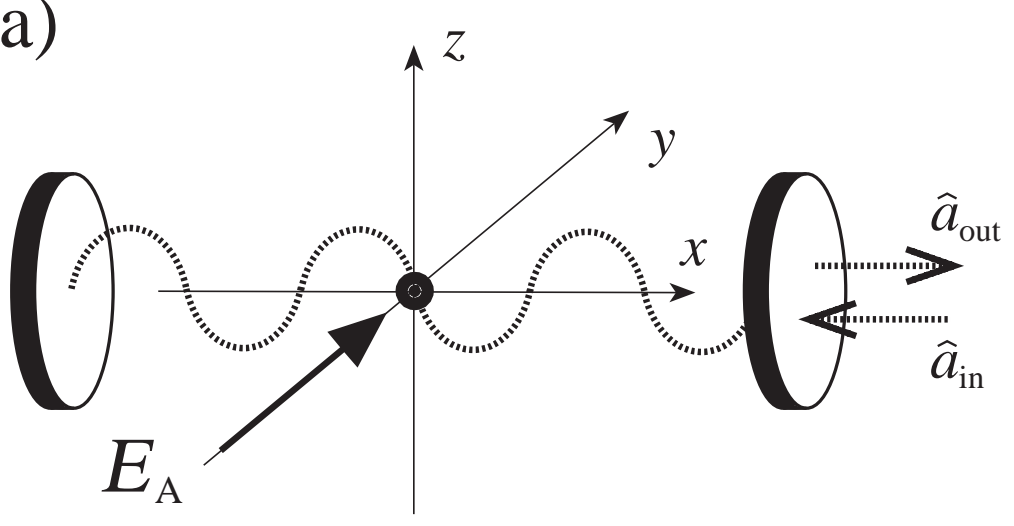

(b)

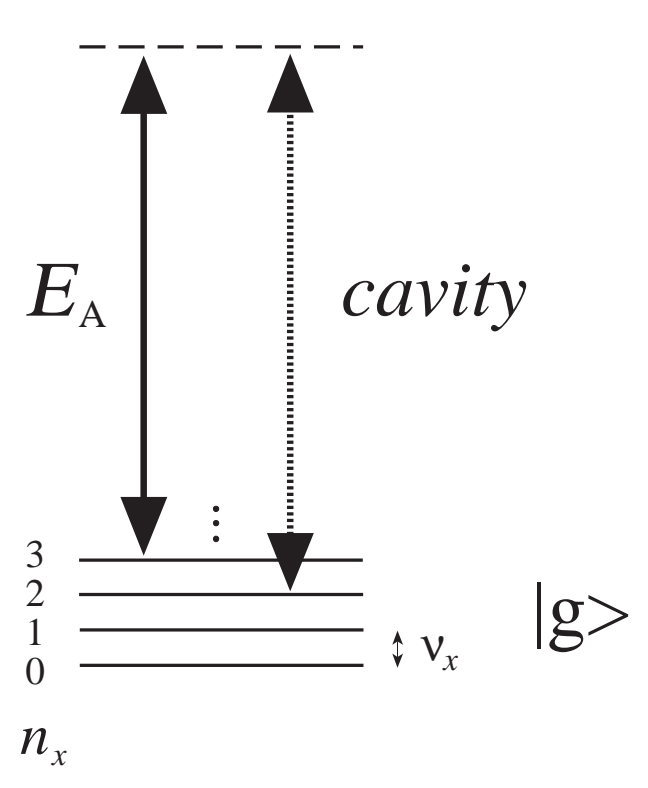

Figure 3 

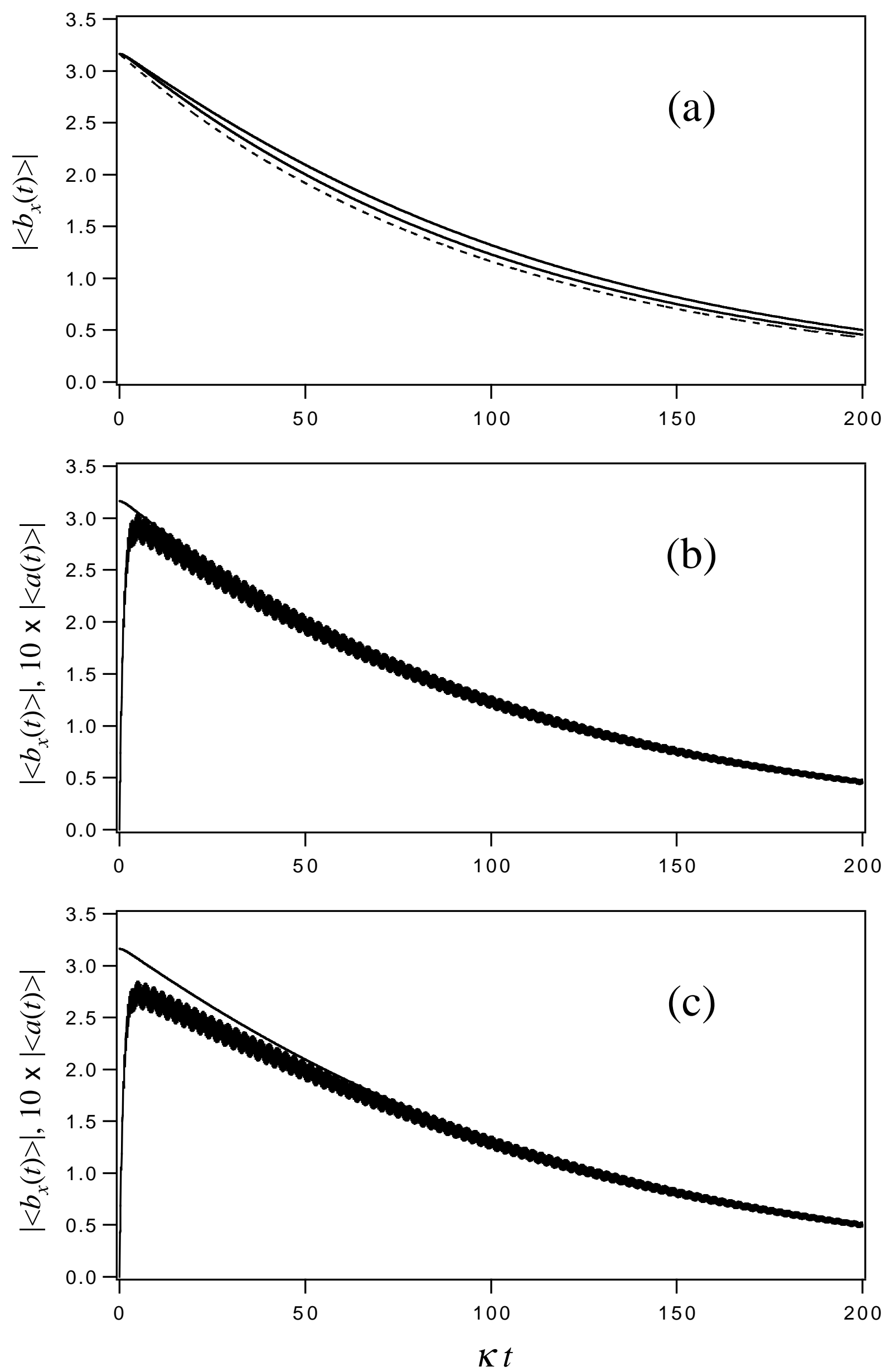

Figure 4 


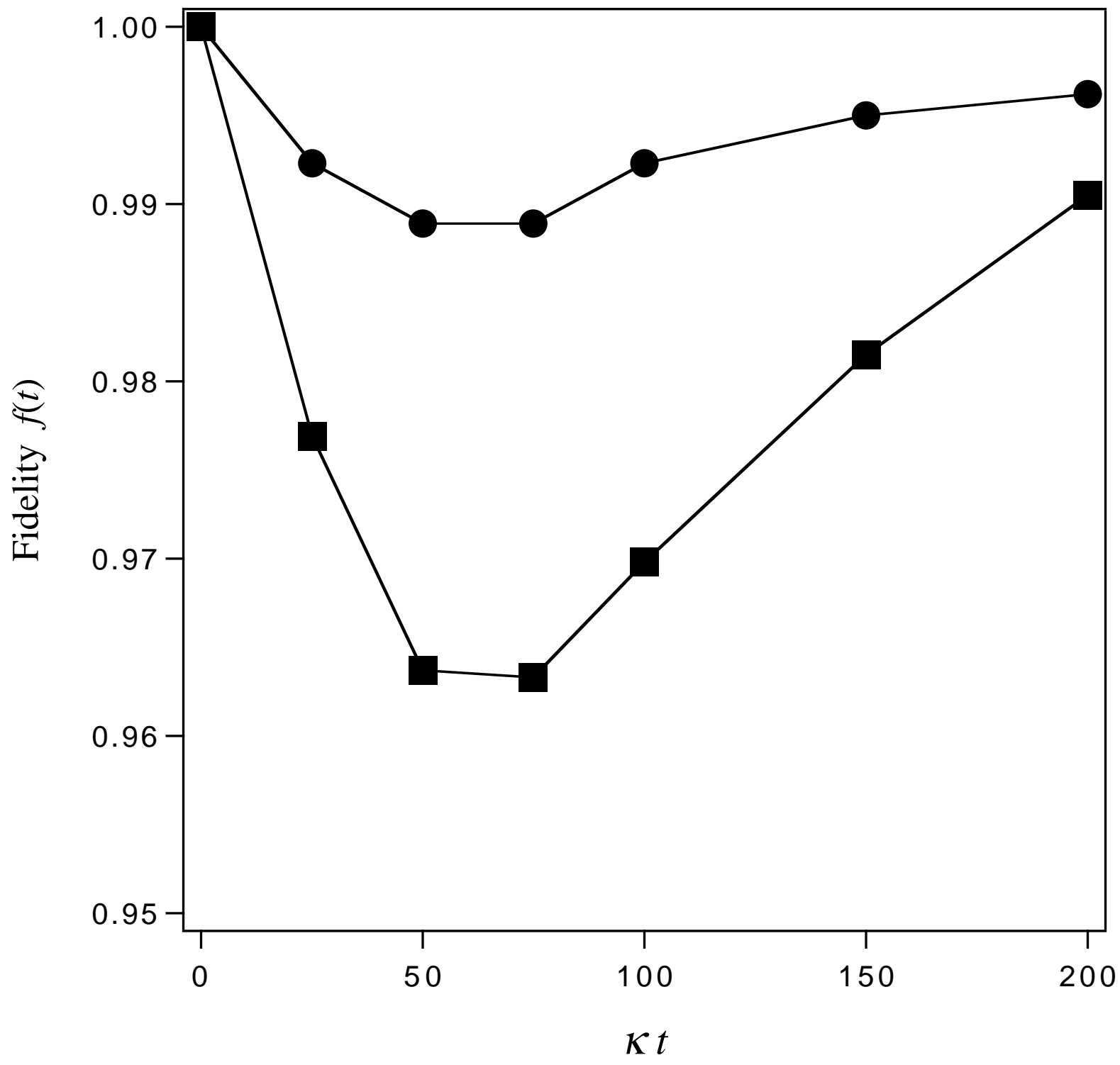

Figure 5 

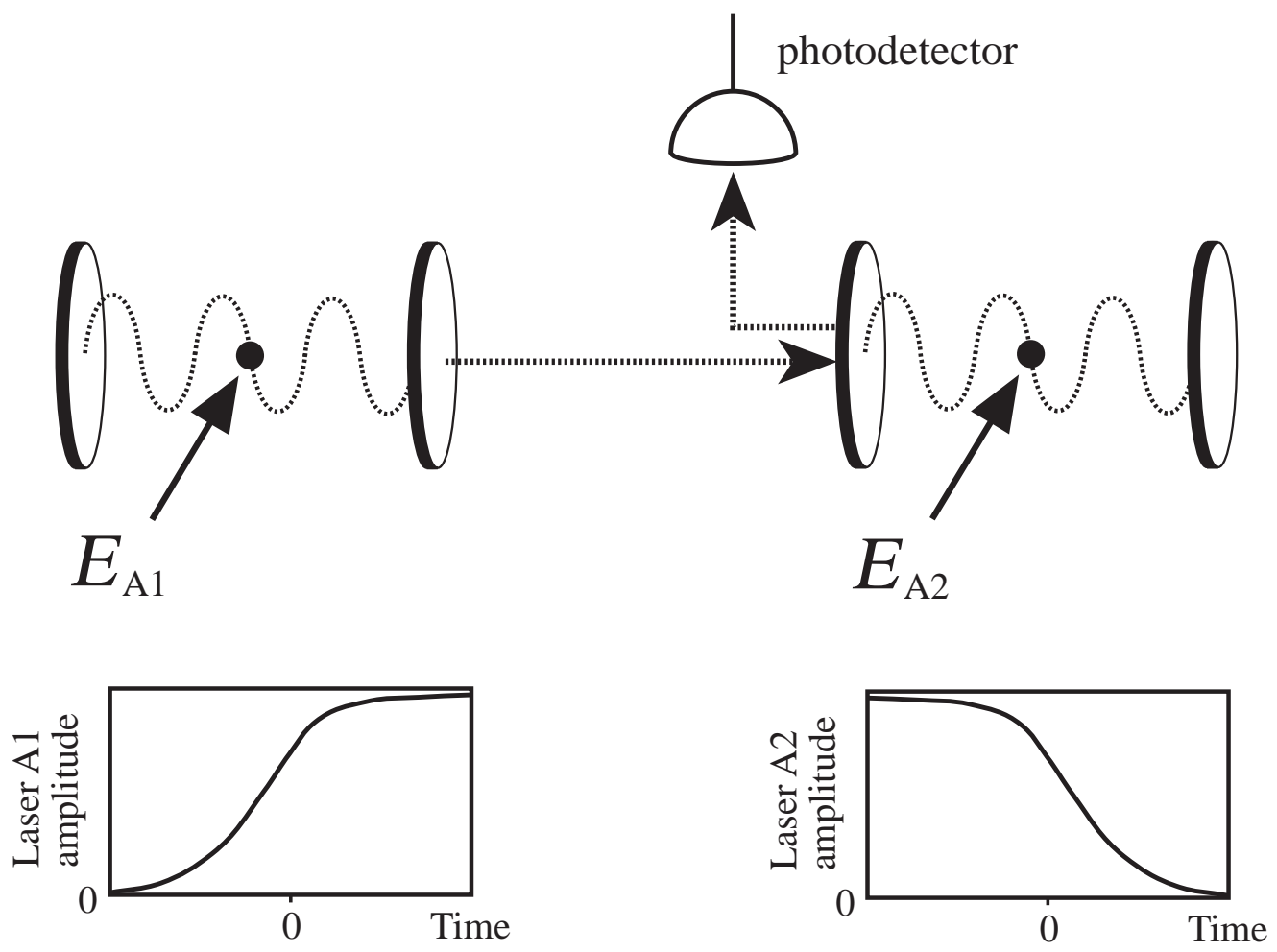

Figure 6 
(a)

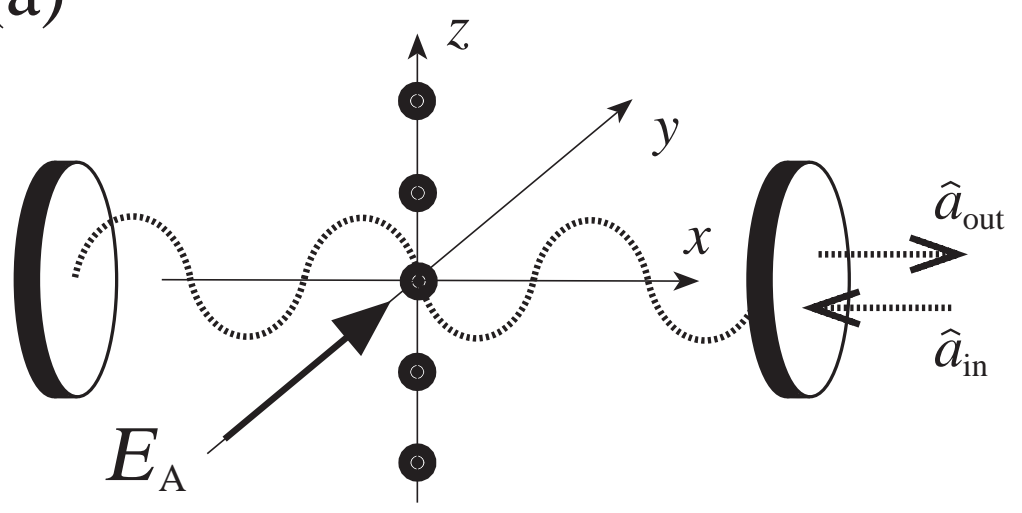

(b)

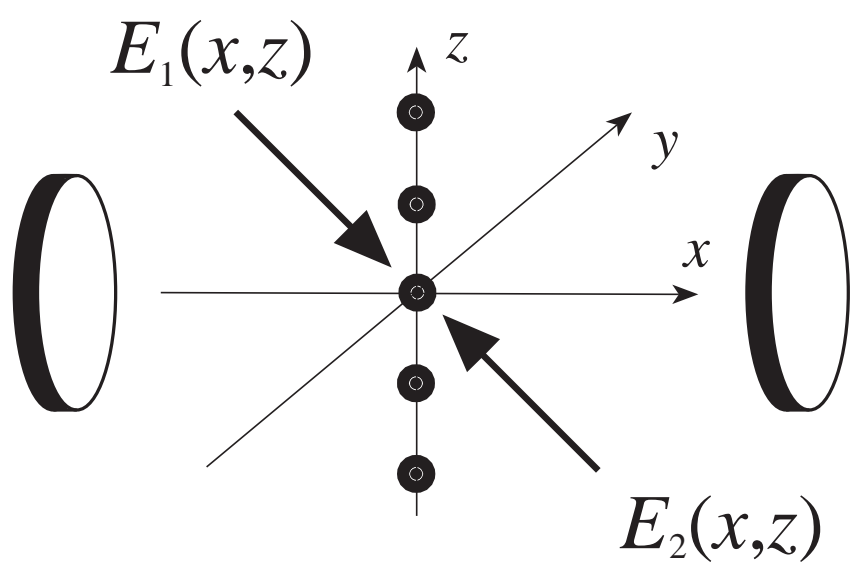

Figure 7 